\title{
EVOLUTIONARY ANALYSIS OF GASEOUS SUB-NEPTUNE-MASS PLANETS WITH MESA
}

\author{
Howard Chen ${ }^{1}$ and Leslie A. Rogers $2,3,4,5,6$ \\ ${ }^{1}$ Department of Physics, Boston University, 590 Commonwealth Avenue, Boston, MA 02215, USA; howardchen2021@u.northwestern.edu \\ ${ }^{2}$ Department of Astronomy, California Institute of Technology, MC249-17, 1200 East California Boulevard, Pasadena, CA 91125, USA \\ ${ }^{3}$ Division of Geological and Planetary Sciences, California Institute of Technology, MC249-17, 1200 East California Boulevard, Pasadena, CA 91125, USA \\ ${ }^{4}$ Department of Earth \& Planetary Sciences, University of California, 307 McCone Hall, Berkeley, CA 94720-4767, USA \\ Received 2015 August 29; revised 2016 August 26; accepted 2016 August 29; published 2016 November 7
}

\begin{abstract}
Sub-Neptune-sized exoplanets represent the most common types of planets in the Milky Way, yet many of their properties are unknown. Here, we present a prescription to adapt the capabilities of the stellar evolution toolkit Modules for Experiments in Stellar Astrophysics to model sub-Neptune-mass planets with H/He envelopes. With the addition of routines treating the planet core luminosity, heavy-element enrichment, atmospheric boundary condition, and mass-loss due to hydrodynamic winds, the evolutionary pathways of planets with diverse starting conditions are more accurately constrained. Using these dynamical models, we construct mass-composition relationships of planets from $1-400 M_{\oplus}$ and investigate how mass-loss impacts their composition and evolution history. We demonstrate that planet radii are typically insensitive to the evolution pathway that brought the planet to its instantaneous mass, composition and age, with variations from hysteresis $\lesssim 2 \%$. We find that planet envelope mass-loss timescales, $\tau_{\text {env }}$, vary non-monotonically with $\mathrm{H} / \mathrm{He}$ envelope mass fractions (at fixed planet mass). In our simulations of young $(100 \mathrm{Myr})$ low-mass $\left(M_{\mathrm{p}} \lesssim 10 M_{\oplus}\right)$ planets with rocky cores, $\tau_{\text {env }}$ is maximized at $M_{\text {env }} / M_{\mathrm{p}}=1 \%$ to $3 \%$. The resulting convergent mass-loss evolution could potentially imprint itself on the close-in planet population as a preferred $\mathrm{H} / \mathrm{He}$ mass fraction of $\sim 1 \%$. Looking ahead, we anticipate that this numerical code will see widespread applications complementing both 3D models and observational exoplanet surveys.
\end{abstract}

Key words: methods: numerical - planets and satellites: atmospheres - planets and satellites: interiors - planets and satellites: physical evolution

\section{INTRODUCTION}

A striking revelation from NASA's Kepler mission is the profusion of sub-Neptune-sized planets discovered with short orbital periods ( $P_{\text {orb }} \lesssim 50$ days) (Borucki et al. 2011; Batalha et al. 2013; Burke et al. 2014; Han et al. 2014; Rowe et al. 2014; Burke et al. 2015; Mullally et al. 2015). Despite the absence of sub-Neptune, super-Earth-sized planets in our Solar System, they are quite ubiquitous in the Milky Way, comprising the majority of planets found by Kepler (Fressin et al. 2013; Petigura et al. 2013b). For inner orbital distances $\lesssim 0.25$ au, the number of sub-Neptune-mass planets exceeds that of Jovian planets by more than a factor of 30 (Howard et al. 2012).

Among the transiting sub-Neptune-sized planets that have measured masses, many of them have mean densities so low that they must have significant complement of light gases (hydrogen and helium) contributing to the planet volume (e.g., Kepler-11c, d, e, f, g Lissauer et al. 2011, 2013). Wolfgang \& Lopez (2015) inferred from the Kepler radius distribution that most sub-Neptunes should have present-day composition of $\sim 1 \% \mathrm{H} / \mathrm{He}$ envelope, under the assumption that all close-in planets consist of rocky cores surrounded by $\mathrm{H} / \mathrm{He}$ envelopes. Considering the sample of Kepler transiting planets with KeckHIRES radial velocity, follow-up (Marcy et al. 2014), Rogers (2015) showed that at planet radii of $1.6 R_{\oplus}$ (and larger) most close-in planets of that size have sufficiently low mean densities that they require a volatile envelope (consisting of $\mathrm{H} / \mathrm{He}$ and/or water). Notably, a large scattering of mass-radius measurements is found in the sub-Neptune, super-Earth-sized

\footnotetext{
5 Hubble Fellow.

6 NASA Sagan Fellow.
}

regime (Marcy et al. 2014), particularly between 1.6 and $4 R_{\oplus}$ (Weiss \& Marcy 2014). Noise in the mass-radius measurements (which often have large error bars) does not account for all of the apparent scatter; there is evidence for intrinsic dispersion in the masses of planets of a specified size (Wolfgang et al. 2015). The intrinsic scatter of small planet mass-radius measurements is an indicator of compositional diversity (e.g., Rogers \& Seager 2010).

The composition distribution of planets observed today reflects both the initial outcomes from planet formation, and subsequent post-formation evolution processes. To study the latter, a useful avenue is numerical simulations of the planets' thermophysical evolution. Stellar flux delays the cooling and contraction of close-in planets. At the same time, higher levels of incident flux also mean greater susceptibility to atmospheric escape. By "backtracking" the thermal and mass-loss evolution history, inferences about the present-day composition and past history of a planet can be made. Such analyses have been carried out for GJ 1214b, CoRoT-7b, and the Kepler 11 and Kepler-36 systems (Valencia et al. 2010; Nettelmann et al. 2011; Lopez et al. 2012; Lopez \& Fortney 2013; Howe \& Burrows 2015).

The effect of atmospheric escape has also been studied for exoplanets in the broader population-level context. For example, the planet mass-loss simulations of Lopez \& Fortney (2013), Owen \& Wu (2013), and Jin et al. (2014) predict a "radius occurrence valley" dividing the sub-populations of close-in planets that have lost/retained their volatile envelopes. Focusing on higher-mass planets, Kurokawa \& Nakamoto (2014) investigated whether mass-loss from hot Jupiters could reproduce the observed "desert of sub-Jupiter-sized exoplanets." More recently, Luger et al. (2015) assessed migration, 
habitability, and transformation of Neptune-like to Earth-like worlds orbiting M-dwarfs.

In this work, we make two contributions. First, we implement several physical formulations related to low-mass planets with hydrogen/helium envelopes into the state of the art Modules for Experiments in Stellar Astrophysics (MESA) code (Paxton et al. 2011, 2013, 2015). MESA is an open source, 1D stellar evolution code that has seen wide use to address problems in stellar astrophysics such as low-mass and high-mass stars, white dwarfs, young neutron stars, and presupernova outbursts or supernova core collapse (e.g., Wolf et al. 2013; Mcley \& Soker 2014; Perna et al. 2014). Only a handful of planetary studies using MESA exist in the literature (Batygin \& Stevenson 2013; Owen \& Wu 2013; Valsecchi et al. 2014, 2015; Jackson et al. 2016), and there is still much potential to push the numerical code to even lower planet masses $\left(1-10 M_{\oplus}\right)$. It is our aim that this paper will serve as a platform for future exoplanetary studies with MESA. The open source nature of MESA allows the astronomical community to readily access the numerical extensions introduced here.

Second, we generate suites of planet evolution simulations to compute mass-radius-composition-age relations for low-mass planets, to quantify how those relations depend on evolution history, and, finally, to explore whether photoevaporation can produce a "favored" planet composition (envelope mass fraction). The numerical models and results obtained are available for public access.

This paper is structured as follows. In Section 2, we describe methods for modeling low-mass planets with MESA. We benchmark are simulations against previously published planet evolution calculations in Section 3. We present our numerical results for the coupled thermal-mass-loss evolution of planets in Section 4, and discuss and conclude in Sections 5 and 6.

\section{MODEL AND APPROACH}

We employ the MESA toolkit (Paxton et al. 2011, 2013, 2015) (version 7623) to construct and evolve thousands of planet models. We consider spherically symmetric planets consisting of a heavy-element interior (comprised of rocky material, or a mixture of rock and ice) surrounded by a hydrogen-helium dominated envelope. The 1D stellar evolution module, MESA star, is adapted to evolve planetary $\mathrm{H} / \mathrm{He}$ envelopes.

In simulating planetary $\mathrm{H} / \mathrm{He}$ envelopes, we employ the default MESA input options, unless otherwise stated. For equation of state (EOS) in planetary conditions, we adopted the hydrogen/helium EOS from Saumon et al. (1995). For the sake of simplicity, we restrict ourselves to solar values of metallicity $Z=0.03$ and helium fraction $Y=0.25$, unless otherwise stated. We used the standard low-temperature Rosseland tables (Freedman et al. 2008) and (Freedman et al. 2014) for visible and infrared opacities. The MESA EOS and opacity tables are further described in Paxton et al. $(2011,2013)$.

MESA already has several useful built-in functions designed for the study of planets (e.g., Becker \& Batygin 2013; Paxton et al. 2013; Wu \& Lithwick 2013, and Valsecchi et al. 2014). Some of these built-in capabilities, however, run into issues when trying to create and evolve small planets. The following sections (Sections 2.1 to 2.4) detail our modifications to the MESA code, and recipes for constructing low-mass planets.

\subsection{Initial Starting Models}

Creating an initial starting model in MESA is a multi-step process. First, we make an initial model (using the create_initial_model option) with a fixed pressure and temperature boundary. We specify these values to be comparable to those in the subsequent evolutionary stages. This technique avoids large discrepancies between the created model and parameters imposed in the evolution phase.

Next, we insert an inert core (using the relax_core option) at the bottommost zone, to represent the heavy-element interior of the planet. We use the models of Rogers et al. (2011) to determine the core radius and bulk density. We consider both a "rocky composition" (70\% silicates and $30 \% \mathrm{Fe})$ and an "icerock mixture composition" $\left(67 \% \mathrm{H}_{2} \mathrm{O}, 23 \%\right.$ silicates and $10 \%$ $\mathrm{Fe}$ ) as options for the heavy-element interior. The addition of an inert core at this stage allows the envelope to be more strongly gravitationally bound in the steps that follow.

In the next step, we rescale the entire planet envelope to the desired total planet mass (using the relax_mass_scale option). Another option to reduce the planet mass is via a mass-loss wind (relax_mass Batygin \& Stevenson 2013).

Finally, after having constructed a model with the desired core mass and envelope mass, we still must quantitatively standardize the initial entropy starting conditions before entering the evolution phase. In each simulation, we take the general approach of "reinflating" planets, to reset the planet evolution.

For high-mass planets $\left(M_{\mathrm{p}} \geqslant 17 M_{\oplus}\right)$, we use an artificial core luminosity to re-inflate the planet envelope until it reaches a specified interior entropy threshold (which depends on mass). In a discrete set of test cases, we determined the maximum entropy $\left(s_{\max }\right)$ to which the planets could be inflated before they reach runaway inflation. We fit a linear function to the maximum entropy data as a function of planet mass, $\left(s_{\max } /\left(k_{\mathrm{B}}\right.\right.$ baryon $\left.\left.^{-1}\right)=2.1662 \log \left(M_{\mathrm{p}} / M_{\oplus}\right)+10.6855\right), \quad$ and use this interpolating function to specify the initial entropy of our high-mass simulated planets. For planets with $M_{\mathrm{p}} \geqslant 17 M_{\oplus}$, the artificial core luminosity deposited at the base of the envelope during the reinflation phase is taken to be three times the surface luminosity (total intrinsic luminosity) of the planet after it is left to passively evolve for 1000 years at the end of the mass-reduction phase.

For low-mass models $\lesssim 17 M_{\oplus}$, special care must be taken when reinflating the planet envelopes. In particular, the procedure used to set the artificial reinflation luminosity in the high-mass regime (three times the planet luminosity at the end of the mass-reduction phase) can blow the envelope of these low-mass loosely bound planets apart. In the low-mass regime, we took a discrete set of test cases (spanning a grid of $M_{\mathrm{p}}$ and $\left.f_{\text {env }}\right)$ and determined in each case the maximum reinflation luminosity factor for which the planet envelope would remain bound (within a precision of 0.02). In practice, this involved finding the highest artificial core luminosity for which the planet radius plateaued to a new equilibrium within $100 \mathrm{Myr}$, instead of expanding to infinity. Then, given any arbitrary planet mass and envelope mass fraction as inputs, we use $2 \mathrm{D}$ interpolation to resolve the specific luminosity.

Once the planet is reinflated, we turn off the artificial reinflation luminosity, and reset the planet age to zero. The zero-age planet model that we have created so far, does not yet account for radiation from the host star. We then use the MESA relax_irradiation option to gradually irradiate our non-irradiated 
model to the desired level. Convergence issues may arise if a high irradiation flux is abruptly introduced on an unirradiated model; not only does the irradiation have to diffuse its way in from the surface of the envelope, but the heat flux escaping from the core has to adjust to new surface boundary conditions. The relax_irradiation option solves this issue by adding a phase in which the flux is turned on slowly.

After the initial starting conditions have been set, we allow the planet to undergo pure thermal evolution (without massloss) for $10 \mathrm{Myr}$ before turning on the mass-loss (following Lopez et al. 2012). These initial cooling periods avoid the runaway mass-loss scenario where the size of planet increases without bound as the simulations enter the mass-loss evolution phases. By the onset of evaporation at $10 \mathrm{Myr}$, models in general should be insensitive to the details of their initial entropy choice. This is because the cooling timescale is typically much less than $10 \mathrm{Myr}$.

\subsection{Atmospheric Boundary Conditions}

When simulating highly irradiated low-mass planets $\left(M_{\mathrm{p}} \lesssim 20 M_{\oplus}\right)$, direct application of MESA's default irradiated atmosphere boundary conditions can lead to problems. In particular, care must be taken to ensure that the atmospheric boundary conditions account for the large changes in surface gravity that the low-mass low-density planets may experience over their evolution as they cool and contract.

In MESA's current gray_irradiated planetary atmosphere option, the surface pressure (at the base of the atmosphere) is resolved at a fixed value. This can lead to issues for simulations of low-mass planets $\left(\lesssim 20 M_{\oplus}\right)$ contracting from very puffy initial states, as the optical depth and opacities at a specified pressure level vary significantly over time. We follow the approach of Owen \& Wu (2013) to overcome this issue in implementing the $T(\tau)$ relation of Guillot (2010) specifying a fixed optical depth $\tau$ (instead of fixed pressure) at the base of the atmosphere. This allows the surface pressure boundary to vary over the course of the planets' evolution. Unless otherwise stated, the planet radii quoted throughout this work, are defined at optical depth $\tau=2 / 3$ (for outgoing thermal radiation).

To relate the irradiation flux absorbed by a planet to the planet's orbital separation and host star properties, a model for the fraction of the irradiation that is absorbed versus reflected by the planet's atmosphere is needed. In general, the Bond albedo of a planet depends on the precise atmospheric composition and the scattering properties of clouds in the planet's atmosphere. Constructing the planet atmosphere directly is beyond the scope of this article, and instead we assume albedo values taken from Fortney et al. (2007).

\subsection{Hydrodynamic Evaporative Mass Loss}

Extreme ultraviolet and X-ray radiation (EUV; $200 \lesssim$ $\lambda \lesssim 911 \AA$ ) from a planet's host star heat the outer reaches of a planet's $\mathrm{H} / \mathrm{He}$ envelope.

For close-orbiting planets, this energy imparted to the atmosphere generates a hydrodynamic wind and causes some gas to escape the planets' gravitational potential well. Similar processes are also proposed to explain the escape of atomic hydrogen in Early Venus and Early Earth (Watson et al. 1981; Kasting \& Pollack 1983). For this study, we implement irradiation-driven mass-loss in MESA using the prescriptions of Murray-Clay et al. (2009). Note that in this work we only focus on implementing hydrodynamic mass-loss; we do not directly treat Jeans escape, direct blow-off, or "photon-limited" escape (Owen \& Alvarez 2016), each of which may be relevant in other regimes.

At low levels of irradiation, the mass-loss is assumed to be energy limited. We follow the energy-limited escape formulation, which was first described by Watson et al. (1981) and then studied by Lammer et al. (2003), Erkaev et al. (2007), Valencia et al. (2010), Lopez et al. (2012), and Luger et al. (2015). The energy-limited mass-loss rate is given by:

$$
\frac{d M_{\mathrm{p}}}{d t}=-\frac{\epsilon_{\mathrm{EUV}} \pi F_{\mathrm{EUV}} R_{\mathrm{p}} R_{\mathrm{EUV}}^{2}}{G M_{\mathrm{p}} K_{\mathrm{tidal}}},
$$

where $\epsilon_{\mathrm{EUV}}$ is the mass-loss efficiency (i.e., the fraction of incident EUV energy that contributes to unbinding the outer layers of the planet), which depends on atmospheric composition and the EUV flux. Here, we adopt a mean efficiency value of 0.1 (Jackson et al. 2012; Lopez et al. 2012), unless otherwise stated. $F_{\mathrm{EUV}}$ is the EUV energy flux from the host star impinging on the planet atmosphere. $R_{\mathrm{p}}$ and $M_{\mathrm{p}}$ are planet radius at optical depth $\tau_{\text {visible }}=1$ (in the visible) and the total mass of the planet respectively. $G$ is the gravitational constant. $R_{\text {Hill }} \approx a\left(M_{\mathrm{p}} / 3 M_{*}\right)^{1 / 3}$ represents the distances outwards to which the planet's gravitational influence dominates over the gravitational influence of the star.

Note that all our models we assume $R_{\text {Hill }}$ to be located well within the exobase where the particle mean free path and atmospheric scale height are comparable. $K_{\text {tidal }}$ (a factor that depends on the ratio of $R_{\mathrm{Hill}}$ and $\left.R_{\mathrm{EUV}}\right)$ corrects for tidal forces, which modify the geometry of the potential energy well and decrease the energy deposition needed to escape the planet's gravity (Erkaev et al. 2007).

Finally, $R_{\mathrm{EUV}}$ is the distance from the center of the planet to the point where the atmosphere is optically thick to EUV photons. To calculate $R_{\mathrm{EUV}}$, which changes with time, we first approximate the difference between $\tau_{\text {visible }}=1$ and $\tau_{\mathrm{EUV}}=1$ (the photoionization base) with

$$
R_{\mathrm{EUV}} \approx R_{\mathrm{p}}+H \ln \left(\frac{P_{\text {photo }}}{P_{\mathrm{EUV}}}\right),
$$

where $H=\left(k_{\mathrm{B}} T_{\text {photo }}\right) /\left(2 m_{\mathrm{H}} g\right)$ is the atmospheric scale height at the photosphere (the factor of 2 in the scale-height equation denotes the molecular form of hydrogen in this regime). $P_{\text {photo }}$ and $T_{\text {photo }}$ are the pressure and temperature at the visible photosphere.

Following Murray-Clay et al. (2009), we estimate the pressure at $\tau_{\mathrm{EUV}}=1$ from the photoionization of hydrogen, $\sigma_{\nu_{0}}=6 \times 10^{-18}\left(h \nu_{0} / 13.6 \mathrm{eV}\right)^{-3} \mathrm{~cm}^{2}$ as $P_{\mathrm{EUV}} \approx\left(m_{\mathrm{H}} G M_{\mathrm{p}}\right) /$ $\left(\sigma_{\nu_{0}} R_{\mathrm{p}}^{2}\right)$, adopting a typical EUV energy of $h \nu_{0}=20 \mathrm{eV}$ instead of integrating over the host star spectrum.

At high EUV fluxes $\left(\gtrsim 10^{4} \mathrm{erg} \mathrm{s}^{-1} \mathrm{~cm}^{-3}\right)$, radiative losses from Ly $\alpha$ cooling become important, mass-loss ceases to be energy limited (Murray-Clay et al. 2009) and a constant massloss efficiency parameter assumption no longer holds. In this regime, photoionizations are balanced by radiative recombinations and radiative losses maintain the temperature of the wind at $T_{\text {wind }} \sim 10^{4} \mathrm{~K}$. This radiation-recombination limited 
mass-loss rate is approximated by,

$$
\begin{aligned}
\left.\frac{d M_{\mathrm{p}}}{d t}\right|_{r r-\lim }= & -\pi\left(\frac{G M_{\mathrm{p}}}{c_{\mathrm{s}}^{2}}\right)^{2} c_{\mathrm{s}} m_{\mathrm{H}} \\
& \times\left(\frac{F_{\mathrm{EUV}} G M_{\mathrm{p}}}{h \nu_{0} \alpha_{r e c} R_{\mathrm{EUV}}^{2} c_{\mathrm{s}}^{2}}\right)^{1 / 2} e^{\left(2-\frac{G M_{\mathrm{p}}}{c_{\mathrm{s}}^{2} R_{\mathrm{EUV}}}\right)},
\end{aligned}
$$

where $c_{\mathrm{S}}=\left(2 k_{\mathrm{B}} T_{\text {wind }} / m_{\mathrm{H}}\right)^{1 / 2}$ is the isothermal sound speed of the fully ionized wind, and $\alpha_{\text {rec }}$ is the radiative recombination coefficient at $10^{4} \mathrm{~K}\left(2.7 \times 10^{-13} \mathrm{~cm}^{3} \mathrm{~s}^{-1}\right)$. Equation (3) is identically Equation (20) of Murray-Clay et al. (2009), but with the dependences on planet mass and surface gravity explicitly preserved (see also Kurokawa \& Nakamoto 2014).

For the EUV luminosity of the planet host star (assumed Sun-like), we adopt the EUV evolution model of Ribas et al. (2005). At each time step, we evaluate both the energy-limited or radiation-recombination-limited mass-loss rates and impose the lesser of the two on the MESA planet model.

\subsection{Heavy-Element Interior}

We incorporate two main updates to MESA to more realistically model planet heavy-element interiors. First, as described in Section 2.1, we use the models of Rogers et al. (2011) to set the core radius and bulk density for a specified heavy-element interior composition and mass.

In this work, we do not model the variations in the core radius as a function of time or pressure over-burden. This assumption is appropriate for the low-mass planets that are the focus of this paper. However, the compression of the planet heavy-element interior due to the pressure of the surrounding envelope starts to be significant for cases in which the pressure at the boundary of the core and envelope exceeds $\sim 10^{10} \mathrm{~Pa}$ (e.g., Mordasini et al. 2012).

Second, we incorporate into MESA a time-varying core luminosity to account for the heavy-element interior's contribution to the envelope energy budget. This contribution may be negligible in hot Jupiters, but is more significant in cases where the core represents a substantial fraction of the total planet mass. For this reason, the energy budget for planets $M_{\mathrm{p}} \lesssim 20 M_{\oplus}$ is more significantly influenced by the presence of a core.

The core luminosity, $L_{\text {core }}$, (i.e., the energy input to the base of the envelope from the heavy-element interior) is commonly modeled as,

$$
L_{\text {core }}=-c_{v} M_{\text {core }} \frac{d T_{\text {core }}}{d t}+L_{\text {radio }}
$$

The first term on the right-hand side of Equation (4) accounts for the thermal inertia of the core. Therein, $c_{\mathrm{v}}$ is the effective constant volume heat capacity of the core (in ergs $\mathrm{K}^{-1} \mathrm{~g}^{-1}$ ), $M_{\text {core }}$ is the mass of the planet's core, and $d T$ core $/ d t$ is time derivative of the effective (mass-weighted) core temperature. We take the Lagrangian time derivative of the temperature at the base of the planet envelope as an approximation to $d T$ core $/ d t$. The most uncertain factor is $c_{\mathrm{v}}$, which could vary depending on the composition of the core and the presence of spatial composition gradients or thermal boundary layers. For the ice-rock core, we adopt a value of $1.2 \mathrm{~J} \mathrm{~K}^{-1} \mathrm{~g}^{-1}$. And the rocky core $1.0 \mathrm{~J} \mathrm{~K}^{-1} \mathrm{~g}^{-1}$ (Guillot et al. 1995). The second term on the right-hand side of Equation (4), $L_{\text {radio }}$, represents the contribution of the decay of radio nuclei to the core luminosity.

$$
L_{\text {radio }}=\chi M_{\text {core }} \sum_{i} H_{\text {initial }, \mathrm{i}} e^{-\lambda_{i} t} .
$$

Above, $\chi$ is the mass fraction of "chrondritic" material in the planet heavy-element interior; this factor is 1 for the Earth-like core composition and 0.33 for the ice-rock core composition. $\lambda_{i}$ is the decay rate constant, and $H_{\text {initial,i }}$ is the initial rate of energy released (per unit mass of rocky material) at $t=0$ by the decay of the $i$ th nuclide. The important long-lived radioactive nuclides are ${ }^{232} \mathrm{Th},{ }^{238} \mathrm{U},{ }^{40} \mathrm{~K}$, and ${ }^{235} \mathrm{U}$ and their half-lives are respectively $1.405 \times 10^{10}, 4.468 \times 10^{9}$, $1.26 \times 10^{9}$, and $7.04 \times 10^{8}$ years. We use the chrondritic abundances and initial energy production rates from Hartmann (2004).

\section{MODEL BENCHMARKING}

Due to the extensive nature of our adaptations to MESA, both in the default parameters and the subroutine modules, there is a need to ensure that our calculations are consistent with those in the current literature. Thus, our numerical results are first presented with a series of benchmarking exercises.

\subsection{Thermal Evolution Benchmarking}

We first simulate the thermal evolution of Jovian and subNeptune planets, without the effects of atmospheric escape.

We run simulations of high-mass $\left(M_{\mathrm{p}} \geqslant 17 M_{\oplus}\right)$ gas giant planets to compare to the models of Fortney et al. (2007) (Table 1). These simulated planet orbit Sun-twins, have heavyelement interiors consisting of $50 \%$ rock and $50 \%$ ice by mass, and have no heating from the core $\left(L_{\text {core }}=0\right.$ ) (as in Fortney et al. 2007).

By ages of $1 \mathrm{Gyr}$, the planet radii predicted by MESA typically agree with the tabulated radii from Fortney et al. (2007) to within 3\% and to better than $7 \%$ for all planet masses and orbital separations. Radius offsets exist at younger ages (100-300 Myr), as would be expected due to the differences in how we set the initial conditions. A crucial difference between our approaches is how we model the absorption of stellar flux in the atmosphere. Fortney et al. (2007) use a grid of selfconsistent radiative-convective equilibrium atmospheric structure models computed following a correlated-K approach, whereas we use a semi-gray atmospheric boundary condition based on the modified $T(\tau)$ relation of Guillot (2010) (Section 2.2). Nonetheless, we find encouraging agreement between MESA and the model planet radii of Fortney et al. (2007).

For planet masses below $20 M_{\oplus}$, we benchmark our results against Lopez \& Fortney (2014) and Bodenheimer \& Lissauer (2014) (Table 2). These simulations have Earth-like rocky cores $(70 \%$ silicate and $30 \% \mathrm{Fe})$ with heat capacity of $1.0 \mathrm{~J} \mathrm{~K}^{-1} \mathrm{~g}^{-1}$ and do not experience the effects of photoevaporation.

At planet masses near $20 M_{\oplus}$ and at low levels of incident irradiation, we find good agreement (within 5\%) between the planet radii predicted by MESA and the results of Lopez \& Fortney (2014). The differences between the two sets of model radii are most extreme at low planet masses and high $f_{\text {env }}$, although all simulations agree to within $20 \%$. The modeled MESA planet radii further show a stronger dependence on the 
Table 1

Planetary Radii Table of Jovian and Sub-Jovian Gas Giants with 50\% Ice-50\% Rock Cores

\begin{tabular}{|c|c|c|c|c|c|c|c|c|c|c|c|c|c|c|}
\hline $\begin{array}{l}\text { Age } \\
\text { (Gyr) }\end{array}$ & $\begin{array}{c}\text { Separation } \\
\text { (au) }\end{array}$ & $\begin{array}{c}\text { Core Mass } \\
\left(M_{\oplus}\right)\end{array}$ & 0.0535 & 0.0881 & 0.115 & 0.242 & 0.406 & 0.676 & 1.0 & 1.46 & 2.44 & 4.07 & 6.78 & 11.31 \\
\hline 0.3 & 0.02 & 0.0 & nan & nan & nan & nan & 15.32 & 14.47 & 14.09 & 14.0 & 14.02 & 14.0 & 13.87 & 13.55 \\
\hline 0.3 & 0.02 & 10.0 & 13.17 & 15.37 & 15.26 & 14.57 & 14.22 & 13.87 & 13.79 & 13.83 & 13.95 & 14.04 & 13.98 & 13.69 \\
\hline 0.3 & 0.02 & 25.0 & nan & 5.12 & 8.28 & 11.95 & 12.79 & 13.15 & 13.33 & 13.54 & 13.8 & 13.98 & 13.98 & 13.74 \\
\hline 0.3 & 0.02 & 50.0 & nan & nan & nan & 8.18 & 10.7 & 11.98 & 12.6 & 13.04 & 13.51 & 13.82 & 13.92 & 13.75 \\
\hline 0.3 & 0.02 & 100.0 & nan & nan & nan & nan & 6.73 & 9.8 & 11.14 & 12.05 & 12.91 & 13.47 & 13.73 & 13.68 \\
\hline 0.3 & 0.045 & 0.0 & nan & nan & nan & nan & 13.56 & 13.28 & 13.14 & 13.14 & 13.19 & 13.16 & 12.94 & 12.53 \\
\hline 0.3 & 0.045 & 10.0 & 9.23 & 11.34 & 11.8 & 12.45 & 12.71 & 12.82 & 12.87 & 12.98 & 13.14 & 13.2 & 13.05 & 12.69 \\
\hline 0.3 & 0.045 & 25.0 & nan & 4.62 & 7.17 & 10.51 & 11.57 & 12.16 & 12.47 & 12.73 & 13.01 & 13.15 & 13.07 & 12.76 \\
\hline 0.3 & 0.045 & 50.0 & nan & nan & nan & 7.5 & 9.84 & 11.14 & 11.81 & 12.28 & 12.76 & 13.02 & 13.03 & 12.79 \\
\hline 0.3 & 0.045 & 100.0 & nan & nan & nan & nan & 6.4 & 9.22 & 10.51 & 11.39 & 12.22 & 12.71 & 12.88 & 12.75 \\
\hline 0.3 & 0.1 & 0.0 & nan & nan & nan & nan & 13.24 & 13.08 & 13.0 & 13.02 & 13.1 & 13.08 & 12.86 & 12.43 \\
\hline 0.3 & 0.1 & 10.0 & 8.25 & 10.41 & 11.02 & 12.02 & 12.42 & 12.63 & 12.73 & 12.87 & 13.05 & 13.11 & 12.96 & 12.58 \\
\hline 0.3 & 0.1 & 25.0 & nan & 4.47 & 6.88 & 10.2 & 11.33 & 12.0 & 12.34 & 12.62 & 12.92 & 13.07 & 12.98 & 12.66 \\
\hline 0.3 & 0.1 & 50.0 & nan & nan & nan & 7.34 & 9.67 & 11.0 & 11.68 & 12.18 & 12.67 & 12.94 & 12.95 & 12.69 \\
\hline 0.3 & 0.1 & 100.0 & nan & nan & nan & nan & 6.33 & 9.12 & 10.41 & 11.3 & 12.14 & 12.63 & 12.8 & 12.66 \\
\hline 0.3 & 1.0 & 0.0 & nan & nan & nan & nan & 12.44 & 12.53 & 12.58 & 12.68 & 12.86 & 12.94 & 12.82 & 12.41 \\
\hline 0.3 & 1.0 & 10.0 & 6.98 & 8.99 & 9.69 & 11.05 & 11.7 & 12.1 & 12.31 & 12.53 & 12.81 & 12.97 & 12.91 & 12.56 \\
\hline 0.3 & 1.0 & 25.0 & nan & 4.23 & 6.35 & 9.51 & 10.73 & 11.52 & 11.94 & 12.29 & 12.69 & 12.93 & 12.94 & 12.64 \\
\hline 0.3 & 1.0 & 50.0 & nan & nan & nan & 6.99 & 9.23 & 10.6 & 11.33 & 11.88 & 12.45 & 12.81 & 12.9 & 12.68 \\
\hline 0.3 & 1.0 & 100.0 & nan & nan & nan & nan & 6.15 & 8.84 & 10.13 & 11.05 & 11.94 & 12.51 & 12.76 & 12.64 \\
\hline 1.0 & 0.02 & 0.0 & nan & nan & nan & nan & 14.1 & 13.61 & 13.37 & 13.33 & 13.36 & 13.34 & 13.14 & 12.73 \\
\hline 1.0 & 0.02 & 10.0 & 11.24 & 13.1 & 13.22 & 13.19 & 13.18 & 13.12 & 13.11 & 13.18 & 13.31 & 13.36 & 13.22 & 12.86 \\
\hline 1.0 & 0.02 & 25.0 & nan & 4.83 & 7.59 & 11.0 & 11.95 & 12.44 & 12.69 & 12.91 & 13.18 & 13.31 & 13.24 & 12.93 \\
\hline 1.0 & 0.02 & 50.0 & nan & nan & nan & 7.72 & 10.1 & 11.37 & 12.0 & 12.45 & 12.91 & 13.18 & 13.2 & 12.96 \\
\hline 1.0 & 0.02 & 100.0 & nan & nan & nan & nan & 6.48 & 9.37 & 10.66 & 11.54 & 12.36 & 12.86 & 13.04 & 12.92 \\
\hline 1.0 & 0.045 & 0.0 & nan & nan & nan & nan & 12.76 & 12.68 & 12.62 & 12.65 & 12.72 & 12.68 & 12.44 & 11.99 \\
\hline 1.0 & 0.045 & 10.0 & 8.2 & 10.14 & 10.67 & 11.58 & 12.01 & 12.26 & 12.38 & 12.52 & 12.68 & 12.72 & 12.54 & 12.14 \\
\hline 1.0 & 0.045 & 25.0 & nan & 4.42 & 6.7 & 9.87 & 10.99 & 11.66 & 12.0 & 12.28 & 12.57 & 12.69 & 12.58 & 12.23 \\
\hline 1.0 & 0.045 & 50.0 & nan & nan & nan & 7.16 & 9.41 & 10.71 & 11.38 & 11.86 & 12.33 & 12.57 & 12.55 & 12.27 \\
\hline 1.0 & 0.045 & 100.0 & nan & nan & nan & nan & 6.21 & 8.91 & 10.16 & 11.02 & 11.82 & 12.28 & 12.42 & 12.25 \\
\hline 1.0 & 0.1 & 0.0 & nan & nan & nan & nan & 12.45 & 12.49 & 12.48 & 12.54 & 12.63 & 12.6 & 12.37 & 11.91 \\
\hline 1.0 & 0.1 & 10.0 & 7.39 & 9.37 & 10.0 & 11.18 & 11.74 & 12.08 & 12.24 & 12.41 & 12.59 & 12.64 & 12.47 & 12.06 \\
\hline 1.0 & 0.1 & 25.0 & nan & 4.27 & 6.44 & 9.59 & 10.76 & 11.49 & 11.87 & 12.17 & 12.48 & 12.61 & 12.5 & 12.15 \\
\hline 1.0 & 0.1 & 50.0 & nan & nan & nan & 7.01 & 9.24 & 10.57 & 11.26 & 11.76 & 12.25 & 12.5 & 12.48 & 12.2 \\
\hline 1.0 & 0.1 & 100.0 & nan & nan & nan & nan & 6.14 & 8.81 & 10.07 & 10.93 & 11.74 & 12.21 & 12.35 & 12.18 \\
\hline 1.0 & 1.0 & 0.0 & nan & nan & nan & nan & 11.76 & 11.98 & 12.08 & 12.19 & 12.34 & 12.38 & 12.24 & 11.87 \\
\hline 1.0 & 1.0 & 10.0 & 6.41 & 8.27 & 8.97 & 10.39 & 11.11 & 11.6 & 11.85 & 12.06 & 12.3 & 12.41 & 12.33 & 12.02 \\
\hline 1.0 & 1.0 & 25.0 & nan & 4.09 & 6.03 & 9.01 & 10.23 & 11.06 & 11.5 & 11.84 & 12.19 & 12.38 & 12.37 & 12.11 \\
\hline 1.0 & 1.0 & 50.0 & nan & nan & nan & 6.72 & 8.86 & 10.2 & 10.93 & 11.45 & 11.97 & 12.28 & 12.35 & 12.15 \\
\hline 1.0 & 1.0 & 100.0 & nan & nan & nan & nan & 5.99 & 8.56 & 9.79 & 10.67 & 11.5 & 12.01 & 12.22 & 12.13 \\
\hline 4.5 & 0.02 & 0.0 & nan & nan & nan & nan & 12.86 & 12.72 & 12.64 & 12.65 & 12.71 & 12.68 & 12.44 & 11.99 \\
\hline 4.5 & 0.02 & 10.0 & 9.43 & 11.03 & 11.32 & 11.79 & 12.1 & 12.3 & 12.4 & 12.52 & 12.68 & 12.71 & 12.54 & 12.13 \\
\hline 4.5 & 0.02 & 25.0 & nan & 4.6 & 6.91 & 10.0 & 11.05 & 11.69 & 12.02 & 12.28 & 12.56 & 12.68 & 12.57 & 12.22 \\
\hline 4.5 & 0.02 & 50.0 & nan & nan & nan & 7.2 & 9.44 & 10.73 & 11.39 & 11.86 & 12.33 & 12.57 & 12.55 & 12.27 \\
\hline 4.5 & 0.02 & 100.0 & nan & nan & nan & nan & 6.2 & 8.9 & 10.16 & 11.02 & 11.82 & 12.28 & 12.41 & 12.24 \\
\hline 4.5 & 0.045 & 0.0 & nan & nan & nan & nan & 11.83 & 11.98 & 12.04 & 12.12 & 12.22 & 12.19 & 11.94 & 11.47 \\
\hline 4.5 & 0.045 & 10.0 & 7.24 & 8.98 & 9.54 & 10.62 & 11.2 & 11.61 & 11.83 & 12.01 & 12.2 & 12.23 & 12.05 & 11.64 \\
\hline 4.5 & 0.045 & 25.0 & nan & 4.23 & 6.24 & 9.16 & 10.3 & 11.07 & 11.48 & 11.79 & 12.09 & 12.21 & 12.1 & 11.74 \\
\hline 4.5 & 0.045 & 50.0 & nan & nan & nan & 6.77 & 8.89 & 10.2 & 10.9 & 11.4 & 11.88 & 12.12 & 12.09 & 11.8 \\
\hline 4.5 & 0.045 & 100.0 & nan & nan & nan & nan & 5.98 & 8.54 & 9.76 & 10.61 & 11.4 & 11.85 & 11.97 & 11.79 \\
\hline 4.5 & 0.1 & 0.0 & nan & nan & nan & nan & 11.57 & 11.81 & 11.91 & 12.02 & 12.14 & 12.11 & 11.88 & 11.41 \\
\hline 4.5 & 0.1 & 10.0 & 6.56 & 8.34 & 8.98 & 10.29 & 10.96 & 11.45 & 11.7 & 11.91 & 12.11 & 12.16 & 11.98 & 11.57 \\
\hline 4.5 & 0.1 & 25.0 & nan & 4.09 & 6.0 & 8.91 & 10.11 & 10.92 & 11.36 & 11.69 & 12.01 & 12.14 & 12.03 & 11.68 \\
\hline 4.5 & 0.1 & 50.0 & nan & nan & nan & 6.64 & 8.75 & 10.08 & 10.79 & 11.31 & 11.8 & 12.05 & 12.02 & 11.74 \\
\hline 4.5 & 0.1 & 100.0 & nan & nan & nan & nan & 5.92 & 8.45 & 9.68 & 10.53 & 11.33 & 11.78 & 11.91 & 11.73 \\
\hline 4.5 & 1.0 & 0.0 & nan & nan & nan & nan & 11.1 & 11.44 & 11.6 & 11.73 & 11.87 & 11.87 & 11.67 & 11.28 \\
\hline 4.5 & 1.0 & 10.0 & 5.86 & 7.6 & 8.29 & 9.77 & 10.53 & 11.1 & 11.4 & 11.62 & 11.84 & 11.91 & 11.77 & 11.44 \\
\hline 4.5 & 1.0 & 25.0 & nan & 3.93 & 5.72 & 8.53 & 9.75 & 10.61 & 11.08 & 11.42 & 11.75 & 11.9 & 11.82 & 11.54 \\
\hline 4.5 & 1.0 & 50.0 & nan & nan & nan & 6.45 & 8.49 & 9.82 & 10.53 & 11.06 & 11.55 & 11.81 & 11.82 & 11.6 \\
\hline 4.5 & 1.0 & 100.0 & nan & nan & nan & nan & 5.83 & 8.28 & 9.47 & 10.31 & 11.1 & 11.56 & 11.71 & 11.6 \\
\hline
\end{tabular}

Note. The top row presents total mass values in $M_{\text {Jup }}$. The planet radii values in $R_{\oplus}$. The specific mass and flux values were chosen for ease of comparison with Fortney et al. (2007). 
Table 2

Planetary Radii Table of Sub-Neptune-Mass Planets with Earth-like Rocky Interiors at $10 \mathrm{Gyr}$

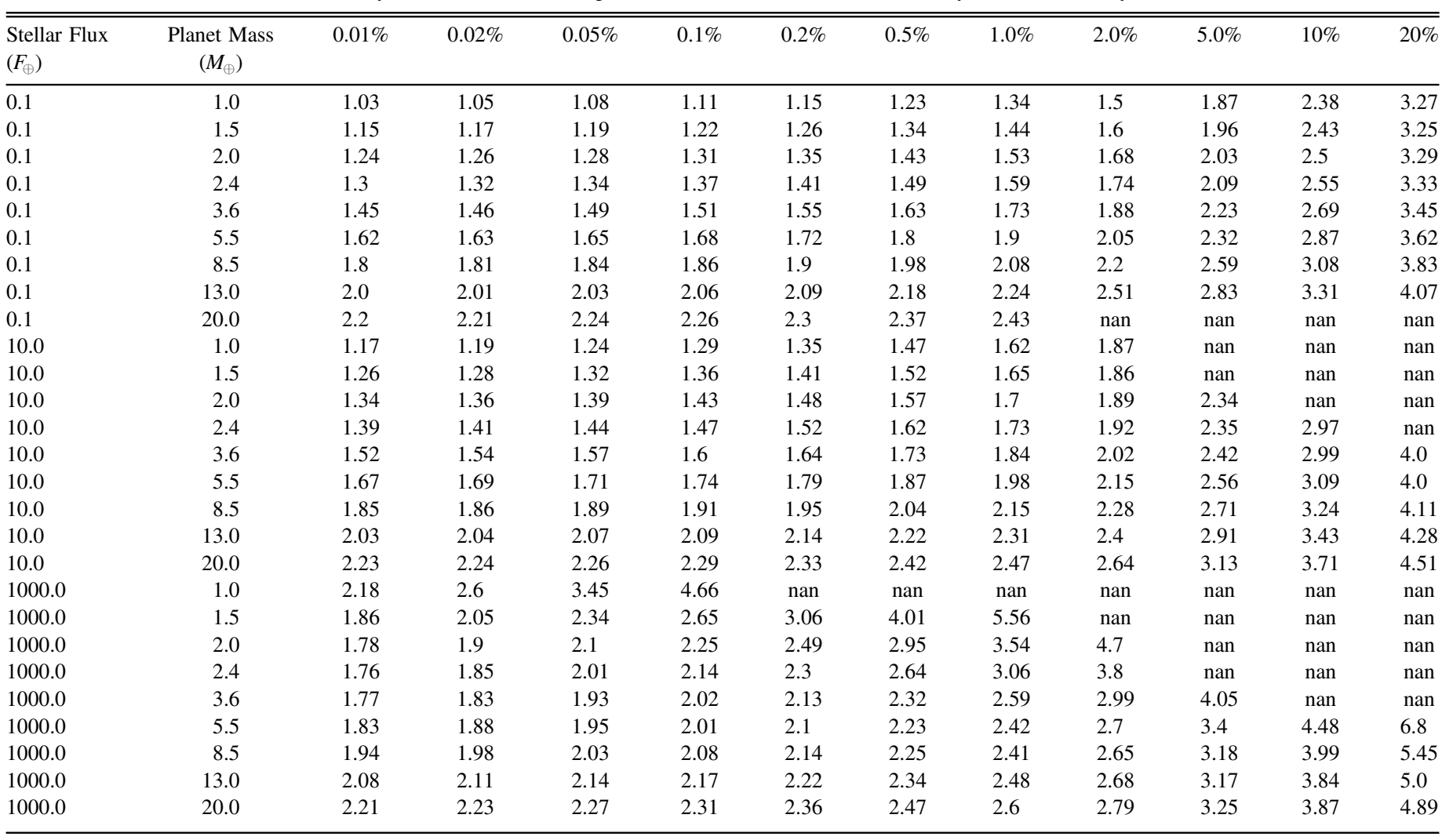

Note. The planet radii values in $R_{\oplus}$. Format and specific mass and flux values are taken for ease of comparison with those from Lopez \& Fortney (2014).

irradiation flux, predicting low-mass planet radii that are larger than Lopez \& Fortney (2014) at $1000 F_{\oplus}$, but smaller than those of Lopez \& Fortney (2014) at $10 F_{\oplus}$.

As for the comparisons with Bodenheimer \& Lissauer (2014), we look at their Table 2 runs with the accretion cutoff times at $2 \mathrm{Myr}$ (runs $2 \mathrm{H}, 1$, and 0.5 ). At $T_{\mathrm{eq}}=500$ and $200 \mathrm{~K}$ and ages of $4 \mathrm{Gyr}$, the final planet radii computed by MESA and by Bodenheimer \& Lissauer (2014) typically agree within $3 \%$ and in all cases agree to better than $10 \%$.

In addition to the Earth-composition heavy-element interiors that are used for benchmarking purposes, we also simulate the same grid of planet masses, envelope mass fractions, and orbital separations with heavy-element interiors that are $70 \%$ ice and 30\% rock by mass (Table 3 ). Note the decisive role that changing the core composition has on the overall planetary radii. As expected, keeping all other parameters identical, the simulated planets with ice-rock cores have larger radii than those with rocky cores. Ice-rock cores have a smaller radio luminosity than rocky cores due to their smaller mass fractions of radioactive nuclides. However, this factor is greatly compensated by the fact that ice-rock cores have lower densities and surface gravities than rocky cores. We shall see that this increase of planet radii with the replacement of icerock heavy-element interiors has important implications for their $\mathrm{H} / \mathrm{He}$ envelope survival rates.

\subsection{Mass-loss Evolution Benchmarking}

In Section 3.1, we modeled planet thermal evolution in the absence of mass-loss and compared the MESA-simulated planet radii to Fortney et al. (2007) and Lopez \& Fortney
(2014) (Tables 1 and 2). Here, we turn to benchmarking our evolution calculations including the full evaporation prescription described in Section 2.3.

We follow Murray-Clay et al. (2009) in calculating the massloss rates (for hot Jupiters). For a $0.7 M_{\mathrm{J}}$ planet with radius 1.4 $R_{\mathrm{J}}$ located at 0.05 au around a solar-mass (G-type) star, they computed a present mass-loss rate of $\sim 4 \times 10^{10} \mathrm{~g} \mathrm{~s}^{-1}$ and a maximum mass-loss rate of $\sim 6 \times 10^{12} \mathrm{~g} \mathrm{~s}^{-1}$. Our evolutionary calculations yield a close result of $5.5 \times 10^{10} \mathrm{~g} \mathrm{~s}^{-1}$ at $4.5 \mathrm{Gyr}$, although our modeled planet radius at this time is only $1.2 R_{\mathrm{J}}$, since we do not include any hot Jupiter inflation mechanisms in our simulations. In addition, these mass-loss rates agree qualitatively well with more detailed calculations including magnetohydrodynamic effects (e.g., Chadney et al. 2015; Tripathi et al. 2015; Vidotto et al. 2015). Our mass-loss rates compared to these studies do not differ by more than an order of magnitude at old ages for planets $\sim 1 M_{\mathrm{J}}$.

We also compared our coupled thermal-mass-loss evolution calculations against previous planet evolution studies. Owen \& $\mathrm{Wu}$ (2013) found that a $318 M_{\oplus}$ planet with a $15 M_{\oplus}$ core at 0.025 au lost $\sim 0.51 \%$ of its total mass over 10 Gyr. In our simulations, a planet with the same mass, composition, and orbital separation lost $5 M_{\oplus}$ (or $\sim 1.81 \%$ of its total mass) over the same time span of $10 \mathrm{Gyr}$. This discrepancy may be attributed to the fact that we use a fixed mass-loss efficiency factor of $\epsilon_{\mathrm{EUV}}=0.1$, whereas Owen \& Wu (2013) perform a more detailed calculation of the mass-loss rate. In performing a full calculation of the hydrodynamics of X-ray driven escape, Owen \& Jackson (2012) concluded that Jovian-mass planets drive the least efficient winds, and that the effective 
Table 3

Planetary Radii Table of Sub-Neptune-Mass Planets with Ice-rock Interiors at $10 \mathrm{Gyr}$

\begin{tabular}{|c|c|c|c|c|c|c|c|c|c|c|c|c|}
\hline Stellar Flux & Planet Mass & $0.01 \%$ & $0.02 \%$ & $0.05 \%$ & $0.1 \%$ & $0.2 \%$ & $0.5 \%$ & $1.0 \%$ & $2.0 \%$ & $5.0 \%$ & $10 \%$ & $20 \%$ \\
\hline 0.1 & 1.0 & 1.36 & 1.38 & 1.41 & 1.45 & 1.49 & 1.58 & 1.68 & 1.83 & 2.19 & 2.62 & 3.51 \\
\hline 0.1 & 1.5 & 1.51 & 1.52 & 1.55 & 1.58 & 1.63 & 1.71 & 1.81 & 1.95 & 2.29 & 2.73 & 3.49 \\
\hline 0.1 & 2.0 & 1.62 & 1.63 & 1.66 & 1.69 & 1.73 & 1.81 & 1.91 & 2.05 & 2.37 & 2.8 & 3.53 \\
\hline 0.1 & 2.4 & 1.69 & 1.71 & 1.74 & 1.77 & 1.8 & 1.88 & 1.98 & 2.12 & 2.44 & 2.86 & 3.57 \\
\hline 0.1 & 3.6 & 1.87 & 1.89 & 1.91 & 1.94 & 1.98 & 2.05 & 2.15 & 2.29 & 2.6 & 3.01 & 3.71 \\
\hline 0.1 & 5.5 & 2.08 & 2.09 & 2.12 & 2.14 & 2.18 & 2.26 & 2.35 & 2.49 & 2.8 & 3.22 & 3.91 \\
\hline 0.1 & 8.5 & 2.31 & 2.32 & 2.35 & 2.37 & 2.41 & 2.49 & 2.58 & 2.72 & 3.03 & 3.47 & 4.15 \\
\hline 0.1 & 13.0 & 2.56 & 2.57 & 2.59 & 2.62 & 2.65 & 2.73 & 2.82 & 2.97 & 3.23 & 3.74 & 4.43 \\
\hline 0.1 & 20.0 & 2.82 & 2.83 & 2.86 & 2.88 & 2.92 & 3.0 & 3.09 & 3.2 & 3.6 & 4.04 & 4.75 \\
\hline 10.0 & 1.0 & 1.58 & 1.63 & 1.7 & 1.77 & 1.85 & 2.0 & 2.17 & 2.44 & nan & nan & nan \\
\hline 10.0 & 1.5 & 1.68 & 1.72 & 1.78 & 1.83 & 1.89 & 2.01 & 2.15 & 2.37 & nan & nan & nan \\
\hline 10.0 & 2.0 & 1.77 & 1.8 & 1.85 & 1.89 & 1.95 & 2.06 & 2.18 & 2.37 & 2.82 & nan & nan \\
\hline 10.0 & 2.4 & 1.83 & 1.86 & 1.9 & 1.94 & 2.0 & 2.1 & 2.22 & 2.4 & 2.82 & 2.97 & nan \\
\hline 10.0 & 3.6 & 1.99 & 2.01 & 2.04 & 2.08 & 2.13 & 2.22 & 2.33 & 2.49 & 2.87 & 3.39 & 4.0 \\
\hline 10.0 & 5.5 & 2.17 & 2.19 & 2.22 & 2.25 & 2.29 & 2.38 & 2.48 & 2.64 & 3.0 & 3.49 & 4.35 \\
\hline 10.0 & 8.5 & 2.38 & 2.4 & 2.43 & 2.46 & 2.5 & 2.58 & 2.68 & 2.83 & 3.18 & 3.66 & 4.47 \\
\hline 10.0 & 13.0 & 2.62 & 2.63 & 2.66 & 2.68 & 2.72 & 2.8 & 2.9 & 3.06 & 3.42 & 3.89 & 4.66 \\
\hline 10.0 & 20.0 & 2.87 & 2.88 & 2.9 & 2.93 & 2.97 & 3.05 & 3.15 & 3.27 & 3.68 & 4.15 & 4.93 \\
\hline 1000.0 & 1.0 & 3.65 & 2.6 & 3.45 & 4.66 & nan & nan & nan & nan & nan & nan & nan \\
\hline 1000.0 & 1.5 & 2.8 & 3.21 & 2.34 & 2.65 & 3.06 & 4.01 & 5.56 & nan & nan & nan & nan \\
\hline 1000.0 & 2.0 & 2.58 & 2.81 & 2.1 & 3.62 & 4.16 & 5.25 & 6.79 & 4.7 & nan & nan & nan \\
\hline 1000.0 & 2.4 & 2.49 & 2.67 & 2.01 & 3.24 & 3.6 & 4.25 & 5.04 & 3.8 & nan & nan & nan \\
\hline 1000.0 & 3.6 & 2.42 & 2.55 & 2.7 & 2.86 & 3.05 & 3.35 & 3.69 & 4.24 & 4.05 & nan & nan \\
\hline 1000.0 & 5.5 & 2.46 & 2.54 & 2.65 & 2.73 & 2.85 & 3.06 & 3.26 & 3.6 & 4.35 & 5.49 & 6.8 \\
\hline 1000.0 & 8.5 & 2.57 & 2.62 & 2.69 & 2.75 & 2.84 & 2.99 & 3.14 & 3.39 & 3.94 & 4.7 & 5.45 \\
\hline 1000.0 & 13.0 & 2.7 & 2.74 & 2.81 & 2.86 & 2.93 & 3.05 & 3.18 & 3.38 & 3.84 & 4.48 & 5.58 \\
\hline 1000.0 & 20.0 & 2.9 & 2.93 & 2.98 & 3.02 & 3.07 & 3.17 & 3.29 & 3.49 & 3.91 & 4.48 & 5.42 \\
\hline
\end{tabular}

Note. The planet radii values in $R_{\oplus}$. Format and specific mass and flux values are identical to those from Table 2 .

approximate escape efficiency for this particular planet scenario is $\sim 0.01$. Assuming $\epsilon_{\mathrm{EUV}}=0.01$ in our simulation, the cumulative mass lost agrees very well with the results of Owen \& Jackson (2012), with our simulation losing $\sim 0.57 \%$ of its total mass.

Lopez \& Fortney (2013) simulated mass-loss from a $320 M_{\oplus}$ Jupiter-mass planet with a $64 M_{\oplus}$ core at 0.033 au (roughly $\left.1000 F_{\oplus}\right)$. They found that the planet lost less than $2 \%$ of its total mass. When we model an identical planet, we find a total of $1.1 \%$ of mass lost over $10 \mathrm{Gyr}$. The fact that we find a lower cumulative mass-loss is to be expected, because Lopez \& Fortney (2013) assume energy-limited escape throughout the entire planet evolution, which would lead to an overestimation of the total mass lost.

For simulations of mass-loss from planets in the subNeptune/super-Earth regime, we compare with Figure 3 in Owen \& Wu (2013). This is a (initially) $20 M_{\oplus}$ planet with a $12 M_{\oplus}$ core. With their X-ray and EUV-driven evaporation scheme, Owen \& Wu (2013) found that the planet ended up with about $13 M_{\oplus}$. We find a slightly higher final mass value of $13.45 M_{\oplus}$. This difference may be partly due to the fact that Owen \& Jackson (2012) treats the difference in EUV and X-raydriven wind scenarios more carefully. We also compute the mass-loss evolution of the Kepler-36 system to reproduce Figure 1 from Lopez \& Fortney (2013) (Figure 1). These simulations start with the initial compositions and masses (Kepler-36c: $f_{\text {env }}=22 \%$, and $M_{\mathrm{p}}=9.41 M_{\oplus}$, Kepler-36b: $f_{\text {env }}=22 \%$, and $M_{\mathrm{p}}=4.45 M_{\oplus}$ ) that Lopez \& Fortney (2013) used in their evolution calculations to match the current properties of the planets inferred by (Carter et al. 2012). Our calculated evolution
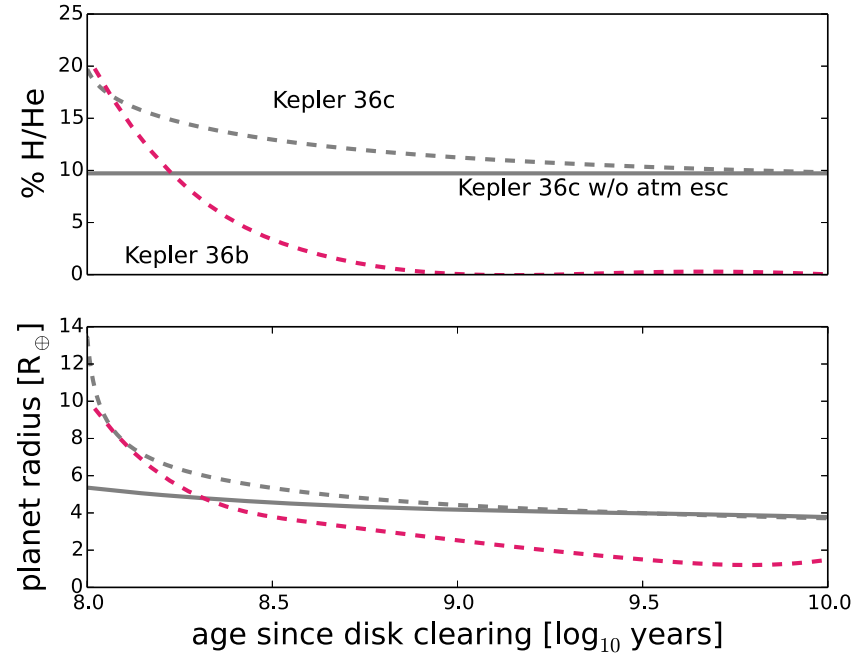

Figure 1. Plot of the thermal/mass-loss evolution of Kepler-36b and c, with radius and envelope mass fraction as a function of time. This plot is designed to be compared with Figure 1 from Lopez \& Fortney (2013). The dashed curves have initial compositions and masses (Kepler-36c: $f_{\text {env }}=22 \%$, and $M_{\mathrm{p}}=9.41 M_{\oplus}$, Kepler-36b: $f_{\text {env }}=22 \%$, and $M_{\mathrm{p}}=4.45 M_{\oplus}$ ) that Lopez \& Fortney (2013) used in their evolution calculations to match the current properties of the planets inferred by (Carter et al. 2012). The solid curve is a simulation that began with the inferred current composition and evolved in the absence of photoevaporation. Note the relatively small final radii difference $(\sim 1.35 \%)$ between solid and dashed curves for Kepler-36c.

tracks of both planets agree qualitatively well with those from Lopez \& Fortney (2013) (Figure 1); we also find that 36b ends with a density higher than $36 \mathrm{c}$ by a factor of 8 , despite the two 
beginning with the same composition. Note, however, that the predicted planet radii in our models are slightly above the measure radii (for instance, we predicted $3.75 R_{\oplus}$ as opposed to the measured $3.67 R_{\oplus}$ for $36 \mathrm{c}$ ).

To summarize Sections 3.1 and 3.2, we find that our planet evolution results are in general agreement with those in the published literature. We also find that the majority of the discrepancies can be attributed to differences in our evaporation schemes and in the way the initial starting conditions are specified.

\section{CALCULATIONS AND RESULTS}

We now turn to applying MESA, with the extensions discussed in Section 2, to simulate the evolution of planets with $\mathrm{H} / \mathrm{He}$ envelopes. We explore some of the important attributes of $\mathrm{H} / \mathrm{He}$-laden planets including ultralow-density configurations, the (in)dependence on evolution history of the current planetary radii and compositions, the role of evaporation in determining planet survival lifetimes as a function of composition, and a synthetic planet population generated by our models.

\subsection{Creating Mass-Radius-Composition Relations with MESA}

Theoretical planetary mass-radius relationships have long been a helpful tool in aiding interpretation and characterization efforts. To produce mass-radius-composition relations (Figure 2), we evolve planets spanning a mass range of $2-20 \mathrm{M}_{\oplus}$, initial envelope mass fractions of $0.05 \%, 0.1 \%$, $0.5 \%, 1 \%, 2 \%, 5 \%, 10 \%, 15 \%, 20 \%$, and $25 \%$, and orbital separations of $0.05,0.075,0.1$, and 1.0 au. We evolve planets with mass-loss for 1 , and $10 \mathrm{Gyr}$ timespans and record both the radii and final compositions of the planets at those ages.

With our adaptations, MESA can simulate $\mathrm{H} / \mathrm{He}$ envelopes surrounding planets down to masses of $1 M_{\oplus}$ and $\mathrm{H} / \mathrm{He}$ mass fractions down to $f_{\text {env }}=1 \times 10^{-6}$, in the absence of $\mathrm{H} / \mathrm{He}$ mass-loss. Below $f_{\text {env }}=1 \times 10^{-6}$ the approximation of an optically thick envelope starts to break down. Typically, it is harder to evolve low-mass $\left(M_{\mathrm{p}} \lesssim 15 M_{\oplus}\right)$ planets as $f_{\text {env }}$ values become greater than $\sim 40 \%$.

With mass-loss turned on in the simulations, there are certain regimes of planet parameter space in which MESA runs into issues, specifically at low planet masses, high $f_{\text {env }}$, and high levels of irradiation. Some of the numerical difficulties can be attributed to the fact that highly irradiated, low-mass, low-density planets are more unstable to mass-loss. For example, an $8 M_{\oplus}$ planet within 0.045 au orbital distance might be unable to hold on to its atmosphere even for a short ( 5000 years) timescale. The time steps in the evolutionary phases are generally much larger than the nominal 1000 years. For this reason, MESA would be unable to resolve the boundary conditions between the two time steps and output convergence errors. For these regimes, instead of losing its $\mathrm{H} / \mathrm{He}$ over $\sim 100 \mathrm{Myr}$, these simulations crash at the start. To study low-mass gaseous planets in extreme equilibrium temperatures, one would need to develop a more suitable boundary condition and initialization parameters. However, these limitations largely do not impede our efforts to create massradius isochrons in our regimes of interest.

From our grid of planet simulations, we derive fitting formulae that may be used to estimate the radius of a planet at specified mass, composition, irradiation flux, and age. Although we advocate interpolating within Tables 2 and 3 (or directly simulating the desired planet using MESA) to derive planet radii for most applications, we provide these
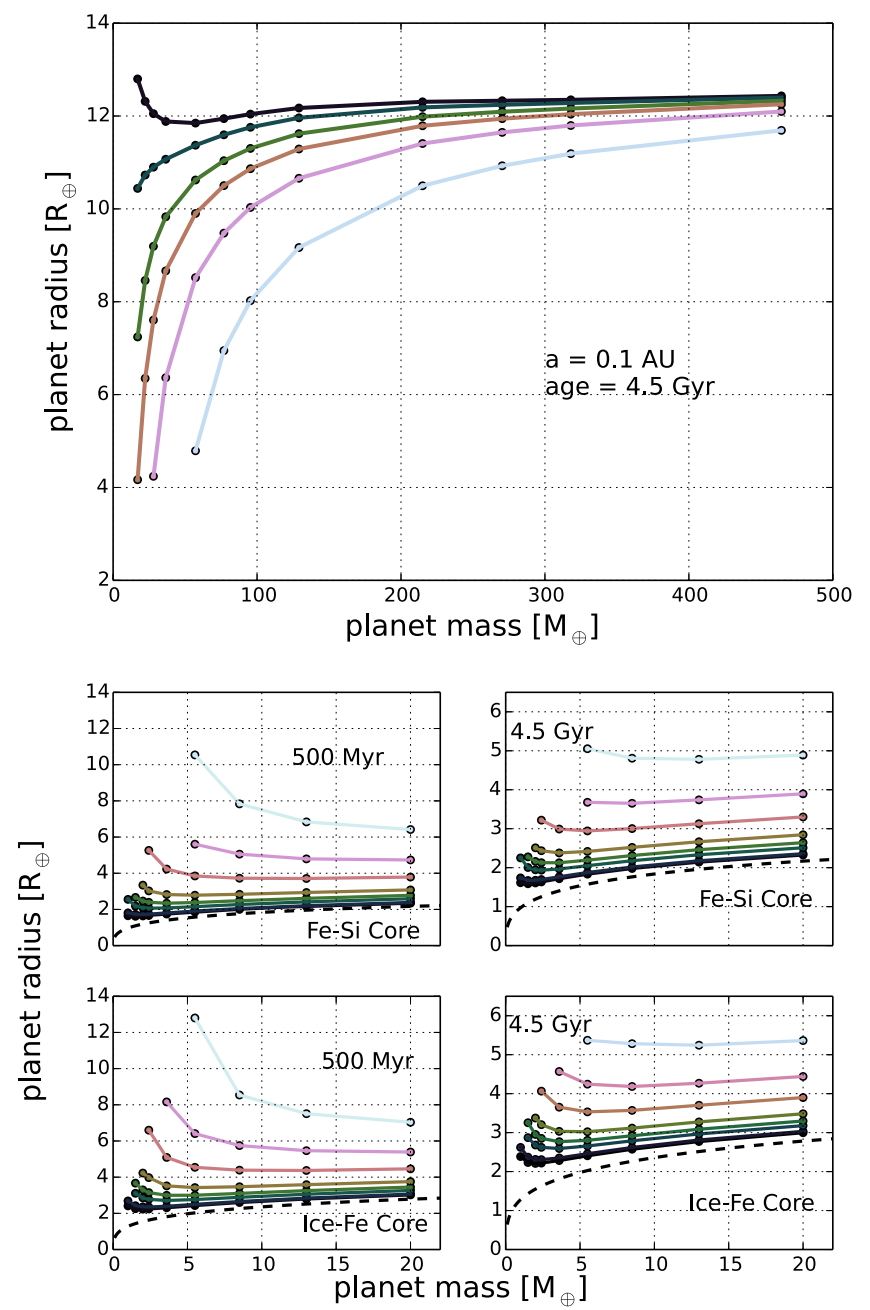

Figure 2. Planetary mass-radius relationship varying core mass or envelope mass fraction in the absence of mass-loss at 0.1 au. For the top panel, the core mass values from top to bottom are $0,5,10,25,50$, and $100 M_{\oplus}$. Notice an "ultralow" density region where Neptune-mass planets attain sizes well above those of Saturn. For the bottom panel, compare particularly between the radii of rocky interior vs. ice-rock-interior simulations. The $f_{\text {env }}=M_{\text {env }} / M_{\text {tot }}$ values from bottom to top are $0.05 \%, 0.1 \%, 0.5 \%, 1.0 \%, 2 \%, 5 \%, 10 \%$, and $15 \%$. A more exhaustive table is included in the Appendix.

fitting formulae for situations where a quick analytic approximation may come in handy. We fit our simulated planet radii to a model in which the logarithm of the contribution of the planet's $\mathrm{H} / \mathrm{He}$ layer to the planet radius $\left(R_{\mathrm{p}}-R_{\text {core }}\right)$ varies quadratically with the logarithms of $M_{\mathrm{p}}, f_{\text {env }}, F_{\mathrm{p}}$, and age

$$
\begin{aligned}
\log _{10}\left(\frac{R_{\mathrm{p}}-R_{\text {core }}}{R_{\oplus}}\right) & =c_{0}+\sum_{i=1}^{4} c_{i} x_{i}+\sum_{i=1}^{4} \sum_{j \geqslant i}^{4} c_{i j} x_{i} x_{j} \\
x_{1} & =\log _{10}\left(\frac{M_{\mathrm{p}}}{M_{\oplus}}\right) \\
x_{2} & =\log _{10}\left(\frac{f_{\text {env }}}{0.05}\right) \\
x_{3} & =\log _{10}\left(\frac{F_{\mathrm{p}}}{F_{\oplus}}\right) \\
x_{4} & =\log _{10}\left(\frac{t}{5 \mathrm{Gyr}}\right) .
\end{aligned}
$$


Table 4

Best fit Coefficients to Analytically Estimate the Radius of a Planet at Specified Mass, Composition, Irradiation Flux, and Age, Using Equation (5)

\begin{tabular}{lrr}
\hline \hline Coefficient & Rocky-Core Planets & Ice-Rock-Core Planets \\
\hline$c_{0}$ & $0.131 \pm 0.006$ & $0.169 \pm 0.008$ \\
$c_{1}$ & $-0.348 \pm 0.008$ & $-0.436 \pm 0.013$ \\
$c_{2}$ & $0.631 \pm 0.003$ & $0.572 \pm 0.005$ \\
$c_{3}$ & $0.104 \pm 0.006$ & $0.154 \pm 0.009$ \\
$c_{4}$ & $-0.179 \pm 0.005$ & $-0.173 \pm 0.007$ \\
$c_{12}$ & $0.028 \pm 0.002$ & $0.014 \pm 0.003$ \\
$c_{13}$ & $-0.168 \pm 0.002$ & $-0.210 \pm 0.003$ \\
$c_{14}$ & $0.008 \pm 0.003$ & $0.006 \pm 0.005$ \\
$c_{23}$ & $-0.045 \pm 0.001$ & $-0.048 \pm 0.001$ \\
$c_{24}$ & $-0.036 \pm 0.001$ & $-0.040 \pm 0.002$ \\
$c_{34}$ & $0.031 \pm 0.002$ & $0.031 \pm 0.002$ \\
$c_{11}$ & $0.209 \pm 0.005$ & $0.246 \pm 0.007$ \\
$c_{22}$ & $0.086 \pm 0.001$ & $0.074 \pm 0.001$ \\
$c_{33}$ & $0.052 \pm 0.002$ & $0.059 \pm 0.003$ \\
$c_{44}$ & $-0.009 \pm 0.002$ & $-0.006 \pm 0.003$ \\
Adjusted $R^{2}$ & 0.993 & 0.987 \\
rms error & 0.035 & 0.047 \\
$R_{\text {core }}$ & $\approx 0.97 M_{\text {core }}^{0.28}$ & $\approx 1.27 M_{\text {core }}^{0.27}$ \\
\hline
\end{tabular}

Note. The radius contribution of the planet envelope is fitted with a quadratic model, $\log _{10}\left(\frac{R_{\mathrm{p}}-R_{\text {core }}}{R_{\oplus}}\right)=c_{0}+\sum_{i=1}^{4} c_{i} x_{i}+\sum_{i=1}^{4} \sum_{j \geqslant i}^{4} c_{i j} x_{i} x_{j}$, where $x_{1}=\log _{10}\left(\frac{M_{\mathrm{p}}}{M_{\oplus}}\right), x_{2}=\log _{10}\left(\frac{f_{\mathrm{env}}}{0.05}\right), x_{3}=\log _{10}\left(\frac{F_{\mathrm{p}}}{F_{\oplus}}\right)$, and $x_{4}=\log _{10}\left(\frac{t}{5 \mathrm{Gyr}}\right)$. These fitting formulae are valid for $10^{-4} \leqslant f_{\text {env }} \leqslant 0.2,1 \leqslant M_{\mathrm{p}} / M_{\oplus} \leqslant 20$, $4 \leqslant F_{\mathrm{p}} / F_{\oplus} \leqslant 400$, and $100 \mathrm{Myr} \leqslant t \leqslant 10 \mathrm{Gyr}$.

We provide the best fitting coefficients in Table 4, both for planets with rocky-composition cores and planets with ice-rock cores. These expressions are valid for $10^{-4} \leqslant f_{\text {env }} \leqslant 0.2$, $1 \leqslant M_{\mathrm{p}} / M_{\oplus} \leqslant 20, \quad 4 \leqslant F_{\mathrm{p}} / F_{\oplus} \leqslant 400, \quad$ and $100 \mathrm{Myr} \leqslant t \leqslant$ 10 Gyr (and should not be extrapolated beyond these ranges). Although we initially endeavored to apply a simpler linear power-law model (as in Lopez \& Fortney 2014), we found nonnegligible curvature in the constant envelope radius hyperplanes in $\log _{10} M_{\mathrm{p}}, \log _{10} f_{\mathrm{env}}, \log _{10} F_{\mathrm{p}}$, and $\log _{10} t$ space, motivating the addition of quadratic terms to the model. Ultimately, these quadratic fits to the rocky-core and ice-rockcore simulations have adjusted $R^{2}$ of 0.993 and 0.987 (compared to 0.914 and 0.816 for the pure power law) and have root mean squared residuals in the envelope radii of 0.0347 and 0.0465 dex (compared to 0.122 and 0.133 dex for the pure power law).

In the above equations, the radius of the planet $\mathrm{H} / \mathrm{He}$ envelope has a steep dependence on the change in envelope fraction and a much shallower dependence on age. This is because non-opacity-enhanced models cool at much higher rates during the first $100 \mathrm{Myr}$ of evolution.

\subsection{The Case of Ultralow-density Planets}

As shown in Figure 2, there exist situations in which simulated sub-Saturn-mass planets have larger sizes than Jupiter. These ultralow-density "puff-ball" planets $\left(\rho \sim 0.2 \mathrm{~g} \mathrm{~cm}^{-3}\right)$ are an interesting new class that challenges planet formation theories. Rogers et al. (2011) showed that moderately irradiated low-mass planets (3-8 $M_{\oplus}$ ) with extended $\mathrm{H} / \mathrm{He}$ envelopes can plausibly have transit radii comparable to Jupiter. Several measurements by Kepler provide evidence for the existence of these extremely low- density planets, for example, Kepler-30d, Kepler-79 and Kepler51 systems (Sanchis-Ojeda et al. 2012; Jontof-Hutter et al. 2014; Masuda 2014).

Batygin \& Stevenson (2013) considered these planets over a wide range of parameter space and calculated their mass-radius isochrons. However, their models assumed a constant core density $\left(\rho_{\text {core }}=5 \mathrm{~g} \mathrm{~cm}^{-3}\right)$ independent of core mass, a luminosity independent of time, and relied on mass-loss timescale arguments to assess the survival of the planet envelope. Self-consistent calculations including a refined thermal-physical model for the planet core and coupled thermal-evaporative evolution are needed, and are now possible with the updates that we have made to MESA. Using these updates, we reproduce the mass-radius figures from Batygin \& Stevenson (2013).

With our coupled thermal-mass-loss simulations (shown in Figure 3), we still find ultralow-density planets (with radii above $10 R_{\oplus}$ and masses below $30 M_{\oplus}$ ), increasing the confidence in the survivability of these planet configurations. With the exception of the simulations with $1 M_{\oplus}$ cores, our simulated planetary radii show good agreement with those from Batygin \& Stevenson (2013).

The main differences between our mass-radius isochrons and those from Batygin \& Stevenson (2013) are threefold. First, our predicted planet radii are generally lower than those in Batygin \& Stevenson (2013). This can be attributed to the different ways in which we set our initial starting conditions. Second, we find that planets over a slightly larger range of planet-mass-core mass-incident flux parameter space are susceptible to losing their entire envelope, with the difference being most pronounced for planets with low-mass cores $\left(\sim 1 M_{\oplus}\right)$. In particular, while a $13 M_{\oplus}$ planet with a $1 M_{\oplus}$ core is expected to survive at $500 \mathrm{~K}$ based on the Batygin \& Stevenson (2013) timescale criterion, such a planet is unstable to mass-loss in our simulations. Third, among the planets that manage to retain their envelopes, we find fewer instances where planet radius increases with decreasing planet mass (at constant core mass). We do still find a negative radius versus mass slope in our $T_{\text {eq }}=700-300 \mathrm{~K}$ simulations with $M_{\text {core }}=1 M_{\oplus}$. However, this rise in radius toward smaller planet masses is less steep than that in Batygin \& Stevenson (2013).

Planets with $d R_{\mathrm{p}} / d M_{\mathrm{p}}<0$ at constant core mass can be susceptible to runaway mass-loss (e.g., Baraffe et al. 2008). In these configurations, the removal of mass from the planet leads to an expansion of the planet radius, and a further increase in the mass-loss rate. This scenario is not treated in Batygin \& Stevenson (2013)'s instantaneous mass-loss timescale criterion. This accounts for why our simulations find a larger area of parameter space excluded by envelope mass-loss in the massradius diagram (Figure 3 ).

\subsection{Assessing Radius as a Proxy for Composition}

Lopez \& Fortney (2014) recasted the mass-radius relations of low-mass planets as more convenient radius-H/He massfraction relations. However, they were focused on models that did not incorporate mass-loss in their evolutionary calculations. Such neglect is appropriate if the primary concern is to assess the effect of stellar irradiation on planetary evolution tracks. With the inclusion of hydrodynamic escape, we reassess some of their main conclusions. 
Figure 2 shows that the radius of a planet with a given $\mathrm{H} / \mathrm{He}$ mass fraction is quite independent of planet mass. This is because at old ages ( $\gtrsim 500 \mathrm{Myr})$, the upturn in the radii of lowmass planets at early times is largely offset by the higher cooling rate. Such striking behavior was previously noted by Lopez \& Fortney (2014), who found that the scatter in the \% $\mathrm{H} / \mathrm{He}$ of their planet models at a specified radius was very low $(\sim 0.3 \mathrm{dex})$. This behavior suggests that planet sizes could act as direct proxy for the bulk $\mathrm{H} / \mathrm{He}$ content. However, the inclusion of mass-loss may complicate matters, because the planet composition does not stay constant, but varies over time.

With the inclusion of mass-loss in our evolution calculations, we find that radius is a proxy for the current planet composition $(\mathrm{H} / \mathrm{He}$ envelope mass fraction).

Figure 4 shows mass-radius contours both at constant initial composition and at constant final compositions for planets at 0.1 au that evolved for $300 \mathrm{Myr}$ and $1 \mathrm{Gyr}$. Despite the addition of mass-loss, the contour lines of constant final composition are flat, with radius largely independent of planet mass $\left(M_{\mathrm{p}} \lesssim 30 M_{\oplus}\right)$ for planets older than $\sim 800 \mathrm{Myr}$. For example, in the mass range of 5-20 $M_{\oplus}$, the radii of our simulated planets with $1 \% \mathrm{H} / \mathrm{He}$ vary by no more than $0.5 R_{\oplus}$, while the radii of planets having $15 \% \mathrm{H} / \mathrm{He}$ vary by no more than $0.1 R_{\oplus}$ over the same mass range. Interestingly, these results hold even at closer orbital separations; at $0.05 \mathrm{au}$, simulations that survive over 1 Gyr (typically $\gtrsim 8 M_{\oplus}$ ) still have flat mass-radius curves. For young planets $\lesssim 500 \mathrm{Myr}$ there is an upturn in the massradius relations (at constant final compositions) at low masses. Radiative cooling over time decreases this "inflation" of lowmass planet sizes to which planets from $1-20 M_{\oplus}$ have comparable radii.

\section{4. (In)Dependence on Evolution History}

Planet evolution calculations, in principle, provide a useful mapping from planet mass, composition, age, and irradiation to planet radius. Often, mass-radius isochrones calculated neglecting mass-loss from $\mathrm{H} / \mathrm{He}$ envelopes (Fortney et al. 2007; Howe et al. 2014; Lopez \& Fortney 2014), are applied even in scenarios in which planetary evaporation processes may be significant. Lopez et al. (2012) previously noted that evaporation only strongly affects thermal evolution in cases of extreme mass-loss when the evaporation timescale becomes comparable to the thermal cooling timescale. However, it is still crucial to more quantitatively calculate the error introduced by not accounting for the full mass-loss history of a planet. In this section, we explore the regimes of parameter space in which planet evolution may exhibit hysteresis, or in other words, in which the radii of planets with identical compositions, masses, and ages depend on their earlier evolution history. We first use the specific case of Kepler-36c to provide an illustrative example of moderate hysteresis.

Figure 1 shows two distinct evolution tracks for Kepler-36c, which both end up at $10 \mathrm{Gyr}$, with identical final compositions and masses $\left(f_{\text {env }}=8.2 \%, M_{\mathrm{p}}=8.01 M_{\oplus}\right)$. The first evolution track (dashed line) includes atmospheric mass-loss over the cumulative history of the planet, and began with an initial composition of $22 \% \mathrm{H} / \mathrm{He}\left(9.41 M_{\oplus}\right.$ total mass). In contrast, the second evolution track (solid line) does not include massloss, staying at constant mass and composition throughout its lifetime. The final planet radii of the two simulations at $10 \mathrm{Gyr}$ show a $\sim 1.35 \%$ difference ( 3.65 versus $3.51 R_{\oplus}$ ), with the nonevaporating model having a smaller radius. While this
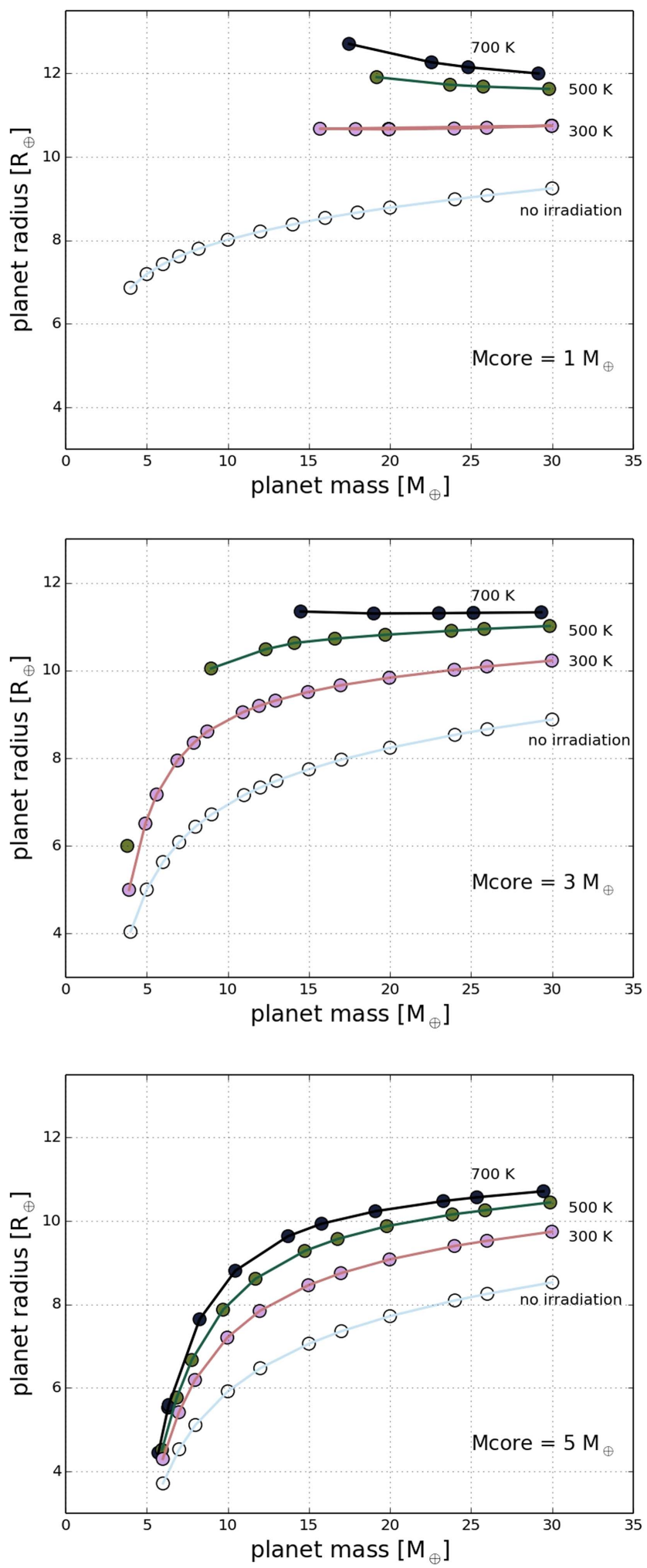

Figure 3. Plot of planet radii vs. mass at $5 \mathrm{Gyr}$, for planets suffering evaporation. The top panel is for planets with $1 M_{\oplus}$ cores, the middle panel is for $3 M_{\oplus}$ cores, and the bottom panel is for $5 M_{\oplus}$ cores. Note that in contrast to the mass-radius relations from the previous sections, these are not for constant composition, but for constant core mass. This is designed to be compared with Figure 3 from Batygin \& Stevenson (2013).

difference in the model radii appears small, it approaches the measurement uncertainty on Kepler-36c's radius $(\lesssim 2 \%)$. This example motivates further investigation to map out the 

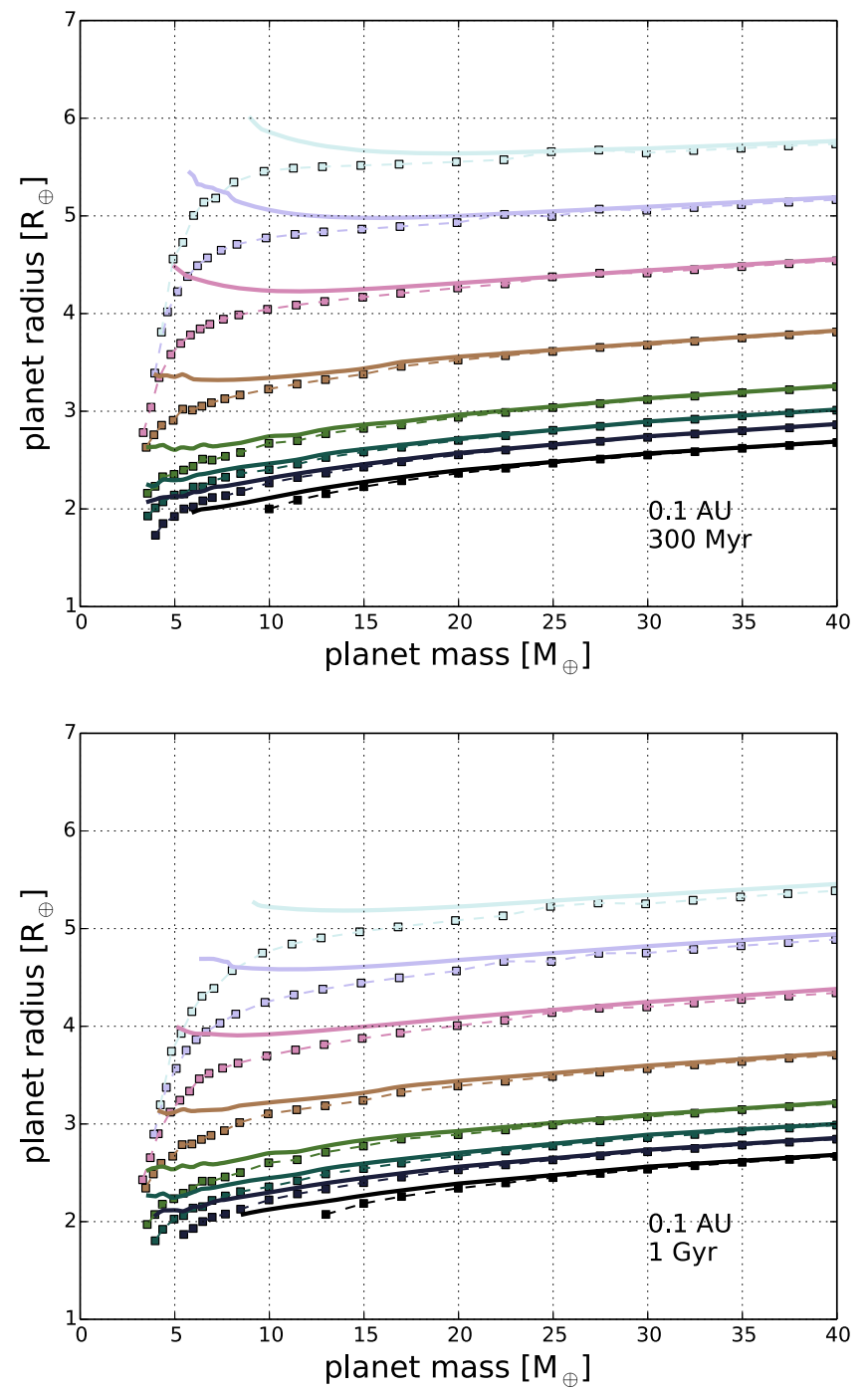

Figure 4. Planetary mass-radius relationships showing contours of constant initial (square markers) and final (solid curves) compositions for models experiencing evaporation. The top panel presents planets that have evolved for $300 \mathrm{Myr}$, while the bottom panel shows planets at $1 \mathrm{Gyr}$. All simulations are presented at an orbital separation of $0.1 \mathrm{au}$. The composition sequence from bottom to top is as follows: note the decisive "flattening" of the radiicomposition curves for constant final composition.

scenarios in which planet radii are even more strongly dependent on the planet evolution history.

We simulate a large grid of planet evolution models to extend the hysteresis experiment to a wider range of planet masses, envelope fractions, and orbital separations. We use the same masses as Section 4.1, initial $f_{\text {env }}=25 \%, 20 \%, 15 \%, 10 \%$, $5.0 \%, 2.0 \%, 1.0 \%, 0.05 \%$, and $0.01 \%$, simulating a grid of evolution calculations with mass-loss turned on. We then performed a second suite of planet evolution simulations with mass-loss turned off. These simulations have compositions identical to those of the mass-losing simulations at 1 and 10 Gyr. The results for 1 Gyr old planets at 0.10 and 0.05 au are shown in Figure 5. In contrast to Section 4.3, where we presented simulations with the same initial compositions, here we compare suites of simulations that begin with different initial compositions, but end up with identical final compositions.

With the inclusion of mass-loss, planets tend to be larger at a specified mass and instantaneous composition. For example, at $0.05 \mathrm{au}$ and $1 \mathrm{Gyr}$ ages, the radius of a planet with a specified instantaneous mass and composition can vary by up to $2.5 \%$ (at $15 M_{\oplus}$, and $f_{\text {env }}=25 \%$ ). At $\sim 0.1$ au, where lower-mass planets $\left(M_{\mathrm{p}} \sim 5 M_{\oplus}\right)$ may retain their atmospheres $(1 \%-10 \%$ $\mathrm{H} / \mathrm{He}$ ), we find overall planet radius differences of $\sim 1 \%$. At a given composition, higher mass-loss rates lead to greater differences in the final sizes. As a corollary, younger simulations receiving higher irradiation with higher envelope mass fractions are marked by a greater difference in planet radii. At older ages beyond $10 \mathrm{Gyr}$, hysteresis is almost nonexistent for planets with $f_{\text {env }} \lesssim 15 \%$.

At lower initial $f_{\text {env }}$ below $10 \%$, the difference between evolution computations including and disregarding mass-loss is never more than $0.5 \%$ regardless of flux received or planet mass. At further-out, orbital semimajor axes of $0.4 \mathrm{au}$, there is minimal discrepancy $(\lesssim 0.01 \%)$ between simulations including and excluding mass-loss in their evolutionary computations. Nonetheless, with the exception of strongly irradiated orbital separations below $\sim 0.06 \mathrm{au}$, the difference is always within the $1 \%$ mark. This suggests that, in most cases the error introduced by using mass-radius isochrons calculated for planets neglecting mass-loss is negligible. Nonetheless, the mass-loss history of a planet can introduce a systematic shift in planet massradius relations that can be comparable to the observations of radius uncertainties for planets with $f_{\text {env }} \gtrsim 10 \%$ at 1 Gyr. This difference typically decreases to between $0.2 \%$ and $0.5 \%$ at even older ages of $10 \mathrm{Gyr}$. The degree of hysteresis is only weakly dependent on planet mass.

To get insight into the reason behind these trends, we can examine the structure of the planet $\mathrm{H} / \mathrm{He}$ envelope. Since more massive planets start with higher interior entropy, removing mass from a planet's envelope leads to an interior structure that is effectively "younger" (higher entropy) than a planet that evolved at a constant lower mass. This explains the fact that the simulated planets that experienced mass-loss have systematically higher radii (at specified mass, age and composition) than the simulated planets that evolved at constant mass. Planets for which the convective $\mathrm{H} / \mathrm{He}$ envelope contributes a significant fraction of the planet radius, should be more susceptible to hysteresis than planets with deep radiative envelopes. The temperature of the outer radiative zone in a planet's $\mathrm{H} / \mathrm{He}$ envelope is set by the radiation incident on the planet from its host star (independent of the cooling history of the planet). It is the location of the radiative-convective boundary and the entropy of the convective zone that may depend on the planet's earlier evolution.

The radiative zone depth in our simulated planets (at specified age and orbital separation) depends only weakly on mass, but more heavily on $f_{\text {env }}$ (Figure 5). The fraction of the envelope radius that is radiative decreases with increasing $\mathrm{H}$ / $\mathrm{He}$ mass fraction. Planets with low $\mathrm{H} / \mathrm{He}$ mass fractions (below $\sim 1 \%$ ) have envelopes that are almost entirely radiative ( $20 \%$ to $100 \%$ of the envelope radius lying in the radiative zone). Planets with very low total mass $\left(M_{\mathrm{p}} \lesssim 7 M_{\oplus}\right)$ also have envelopes that are almost entirely radiative. At the other extreme, comparison of the top and bottom panels in Figure 5 indicates that in regimes where the simulated planets do exhibit hysteresis (at $f_{\text {env }} \gtrsim 10 \%$, where the eventual planet radii differ by $\sim 0.5 \%-1.5 \%$ ), they generally have more substantial convective regions, accounting for at least $30 \%$ of the total radial extent of the planet envelope.

It is possible, however, that the cause of the apparent hysteresis in our planet evolution simulations is numerical in 

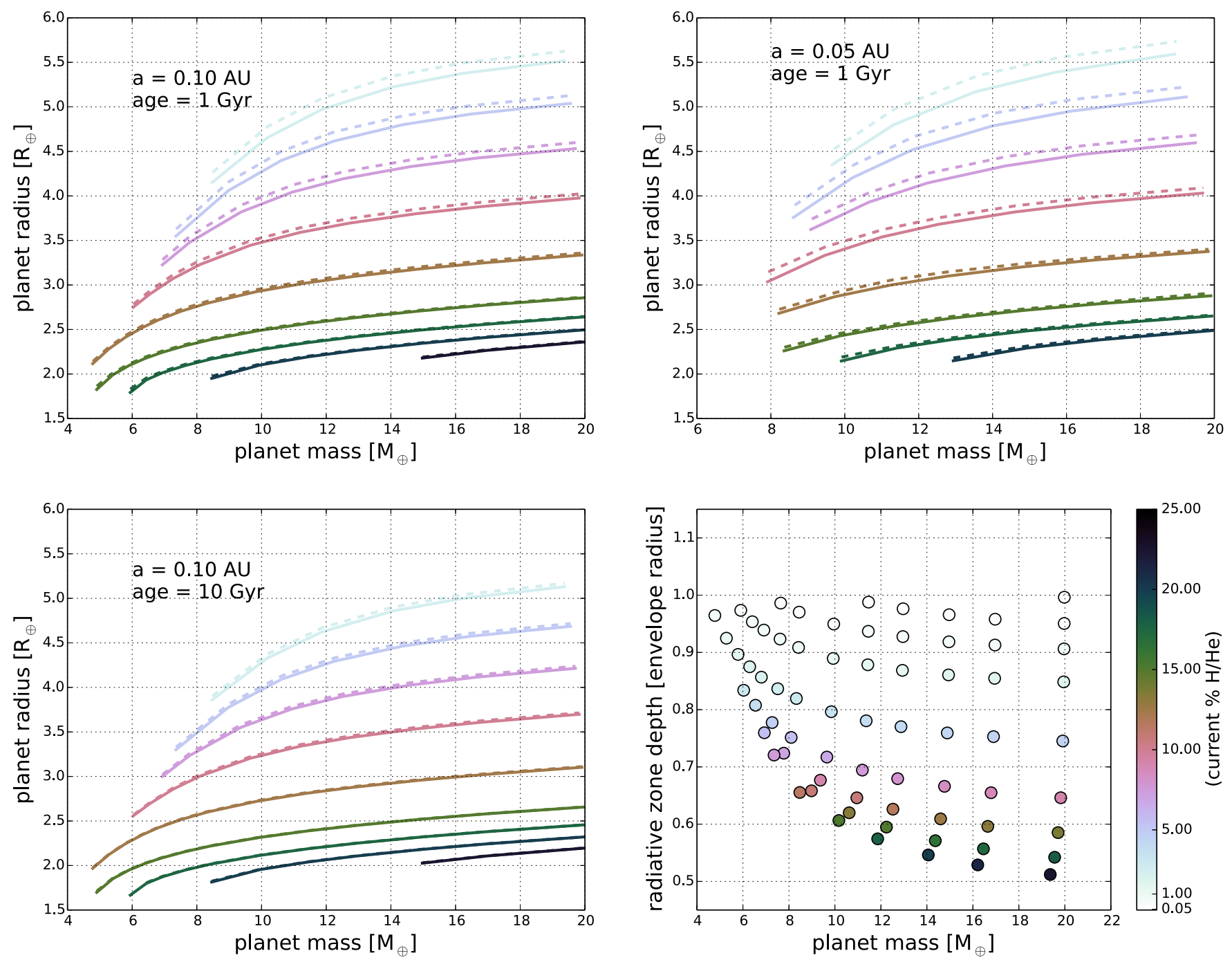

Figure 5. Quantifying the degree of hysteresis with planet evolution tracks experiencing mass-loss. The top panels show a plot of planet mass-radius relations with varying initial envelope mass fractions, at an age of $1 \mathrm{Gyr}$, and at orbital distances of 0.10 au (top left) and 0.05 au (top right). Dotted lines represent simulations of planets experiencing mass-loss, while the solid curves represent planets (of identical instantaneous mass and composition) that evolved at constant mass. The initial envelope mass-fraction values, from top to bottom, are: $25 \%, 20 \%, 15 \%, 10 \%, 5.0 \%, 2.0 \%, 1.0 \%, 0.05 \%$, and $0.01 \%$. Accompanying higher values of $\% \mathrm{H} / \mathrm{He}$ in the planet composition is the increase in the discrepancy between the dotted and solid lines. This discrepancy is weakly dependent on planet mass, with lower-mass simulations $\left(M_{\mathrm{p}} \lesssim 10 M_{\oplus}\right)$ having more significant discrepancy (up to $3 \%$ in planet radius). Conversely, in the regime of low $\mathrm{H} / \mathrm{He}$ mass fractions, there is little difference between the radii of planets following the two different evolution pathways. The bottom left panel shows the same relations at 0.1 au, but for models with the same instantaneous $\mathrm{H} / \mathrm{He}$ fractions at $10 \mathrm{Gyr}$. The bottom right panel presents the fractional radiative zone depth (in units of the total radial thickness of the planet $\mathrm{H} / \mathrm{He}$ envelope, $R_{\text {env }}$ ) as a function of planet mass, for $1 \mathrm{Gyr}$ old planets at 0.1 au. Note how the outer radiative zone represents a larger fraction of the envelope radius at lower $\mathrm{H} / \mathrm{He}$ mass fractions and smaller planet masses, explaining the relative small display of hysteresis in this region of parameter space.

nature. This is because our modified $T(\tau)$ atmospheric boundary condition does not conserve energy globally to machine precision. Since mass-loss has some $p d V$ work associated with it in the upper atmosphere, a fixed temperature boundary condition does not treat this correctly, and in some cases will lead to more energy input into the planet's atmosphere. In reality, however, the advection due to evaporation carries some extra thermal energy out of the planet, thereby enhancing cooling. For the "normal" EUV evaporation, the expectation is that this is a small effect, hence using a fixed $T(\tau)$ relation would not introduce significant errors. However, once the escape enters the "boil-off" regime (e.g., Owen \& Wu 2016), then our current boundary condition would not be suitable.

Regardless of the root cause, (numerical or physical in nature), our main conclusion is unchanged. The error introduced by using mass-composition-radius isochrons calculated for planets neglecting mass-loss is typically small $(\lesssim 1 \%$ in planet radius).

\subsection{A Favored \% H/He Mass Fraction?}

Based on the analysis of the radius distribution of a subset of Kepler planet candidates, Wolfgang \& Lopez (2015) found a typical $\mathrm{H} / \mathrm{He}$ mass fraction of $0.7 \%$ (with a standard deviation of $\sim 0.6$ dex). Ultimately, the compositions of planets observed today are consequences of both the initial formation and subsequent evolution. What role might evaporative planet mass-loss play in producing this $0.7 \%$ typical $\mathrm{H} / \mathrm{He}$ envelope mass fraction?

Our simulations of the coupled thermal and mass-loss evolution of low-mass planets show evidence for a non- 
monotonic relation between planet composition and envelope survival rate. Similar behavior has also been noted in previous planet evaporation parameter studies (Lopez \& Fortney 2013; Jin et al. 2014). In Figure 4, we see that, by the age of $1 \mathrm{Gyr}$, low-mass planets $\left(\lesssim 6 M_{\oplus}\right)$ with high $(\sim 20 \%)$ or very low $(\sim 0.05 \%-0.1 \%)$ envelope mass fractions tend to have lower survival rates. Envelope mass-fraction values in the "intermediate" range seem to have a greater probability of survival. This can be seen in Figure 4 by the fact that the mass-radius curves for $f_{\text {env }}=0.05 \%-2 \%$ extend to lower masses than the mass-radius curves of both higher and lower envelope fractions. This could provide a mechanism to imprint a preferred envelope mass on the planet population.

To more quantitatively examine the situation, it is illuminating to consider the planet envelope mass-loss timescale, defined as $\tau_{\text {env }}=M_{\text {env }} / \dot{M}_{p}$ (e.g., Rogers et al. 2011; Batygin \& Stevenson 2013). This quantity provides an instantaneous measure of how long a planet envelope could "survive" at its current mass-loss rate. For this experiment, we calculate $\tau_{\text {env }}$ at early ages of 100 Myr. We present, in Figure 6, envelope massloss timescales for planets at $0.1 \mathrm{au}$.

In our simulations of planets with rocky cores, the mass-loss timescale (for specified planet mass) is maximized at an intermediate value of $f_{\text {env }}=1 \%-2 \%$. At smaller envelope mass fractions $\left(f_{\text {env }}=0.05 \%\right.$ and $0.1 \%$ ), lifetimes are shorter, because there is less envelope to lose. At higher envelope mass fractions above 5\%, planet radii increase the mass-loss rate as the energy-limited escape is sensitive to the cross-sectional radius (energy-limited $\propto R_{\mathrm{p}}^{3}$ ). Furthermore, planets that lose a significant fraction of their total mass early on would likely completely evaporate before reaching the age of $1 \mathrm{Gyr}$ (Lopez et al. 2012). This may lead to convergent evolution behavior, where planets end up with similar final envelope mass fractions (near where the mass-loss timescale is maximized) for a wide range of initial envelope mass fractions. This trend in our results also indicates that planets with $\sim 1 \% \mathrm{H} / \mathrm{He}$ may "linger" longer at the composition, compared to other values of $f_{\text {env }}$. Once planets with high initial $\mathrm{H} / \mathrm{He}$ fractions reach this $\sim 1 \%$ value, they typically retain similar values of envelope fraction for $\gtrsim 5$ Gyr.

Upon closer examination of the top panel in Figure 6, the planet envelope mass fraction at which the mass-loss timescale is highest increases with planet mass. Below $12 M_{\oplus}$, the simulated planets with $1 \% \mathrm{H} / \mathrm{He}$ mass fraction maximize $\tau_{\text {env }}$. Above $12 M_{\oplus}$, the value of $f_{\text {env }}$ that maximizes $\tau_{\text {env }}$ switches to $5 \%-6 \%$. This hints that "fixed point" compositions to which planets converge may depend on the planet mass, potentially providing an observational diagnostic.

Recall, though, that these $\tau_{\text {env }}$ values correspond to the stellar fluxes at $0.1 \mathrm{au}$. At further-out orbital distances $(\gtrsim 0.1 \mathrm{au})$, the entire distribution shifts upward (toward higher $\tau_{\text {env }}$ ). Conversely, at a closer-in distance, the envelope mass-loss timescales decrease across all values of planet masses, largely preserving the general shape of the distribution.

For planets with lower-density ice-rich cores (lower panel, Figure 6), we see a distinct upward shift in the value of $f_{\text {env }}$ that maximizes $\tau_{\text {env }}$ compared to planets with higher-density rocky cores. The icy-interior models do not generate the same $1 \%$ signature, but instead favor a higher set of "most survivable" envelope mass-fraction values (namely in the $\gtrsim 5 \%$ range for planets $\gtrsim 5 M_{\oplus}$ ). This shift is a consequence of how a lowerdensity heavy-element interior distorts the planet $R_{\mathrm{p}}-f_{\text {env }}$
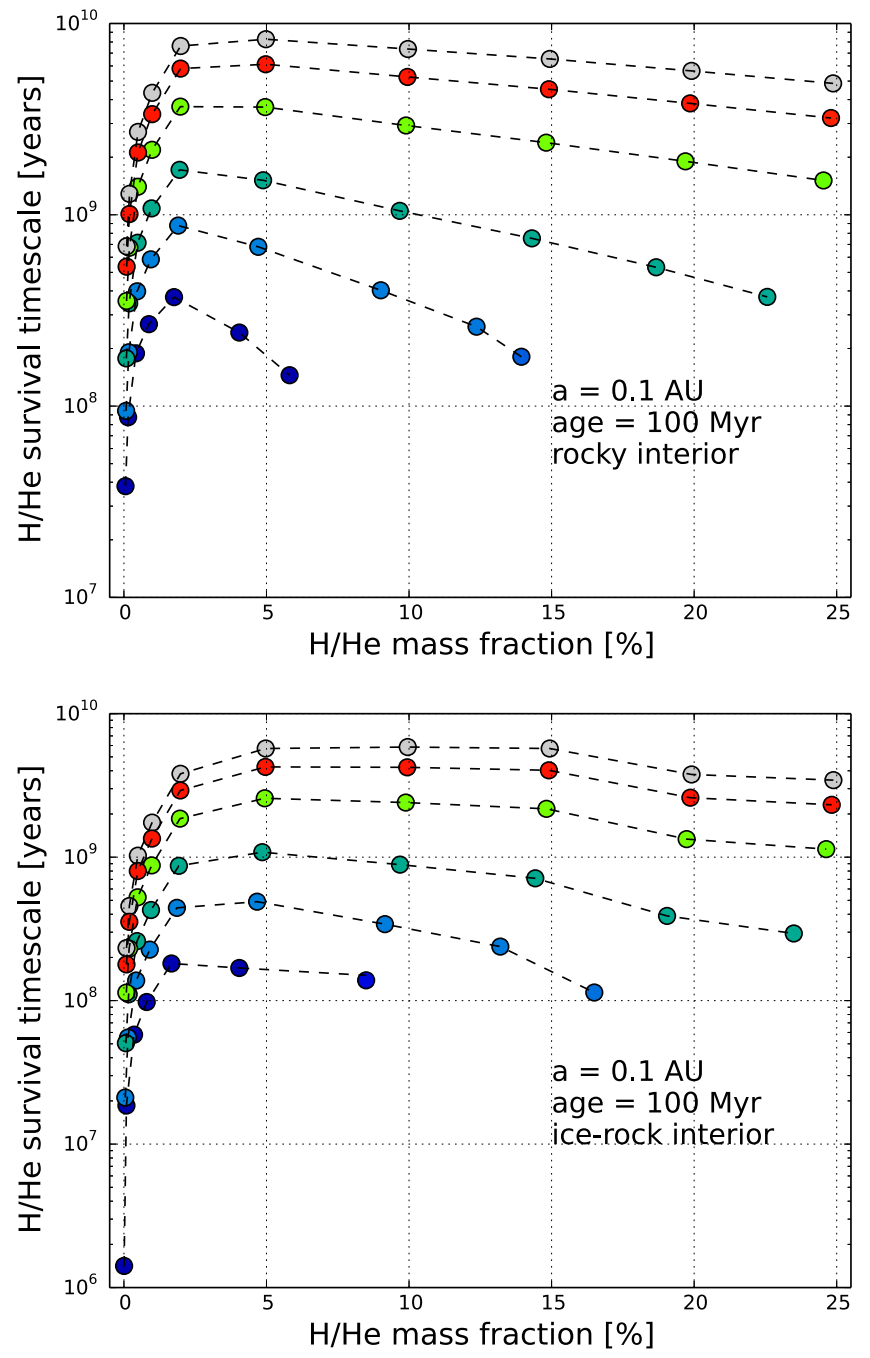

Figure 6. Plot of instantaneous envelope mass-loss timescales, $\tau_{\text {env }}$, as a function of $\mathrm{H} / \mathrm{He}$ fraction and planet mass at 0.1 au. These simulations have Earth-like rocky (top panel) or ice-rock (bottom panel) heavy-element interiors, and solar metallicity envelopes. The initial planet masses for the scatter points from bottom to top are 4.0 (blue), 6.0 (light blue), 8.0 (teal), 13 (green), 17 (red), and 20 (white) $M_{\oplus}$ respectively. The $\tau_{\mathrm{env}}$ values presented are instantaneous mass-loss rates at early times (100 Myr) when the stellar EUV is high. We see evidence of non-monotonic behavior of $\tau_{\text {env }}$ with $f_{\text {env }}$. At this instant, the most "survivable" envelope fraction (at which $\tau_{\text {env }}$ is maximized) is between $1 \%$ and $5 \%$.

relation. At masses below $\sim 13 M_{\oplus}$, the ice-rock interior planets with $5 \%-15 \%$ by mass $\mathrm{H} / \mathrm{He}$ envelopes all have similar trends and near maximal values of $\tau_{\text {env }}$. At yet higher planet masses (above $\sim 13 M_{\oplus}$ ), the $\sim 15 \% \mathrm{H} / \mathrm{He}$ composition takes over as the composition maximizing $\tau_{\text {env }}$. Compared with the rockycore models, this take-over point occurs at a much lower planet mass.

A major caveat in this study of $\tau_{\text {env }}$ is that even if the $\mathrm{H} / \mathrm{He}$ envelope mass fractions of 1\%-5\% surrounding rocky cores maximize the mass-loss timescale at $100 \mathrm{Myr}$, we have not yet established whether this could have a substantial effect on the planet population, specifically whether this effect could plausibly reproduce the $\mathrm{H} / \mathrm{He}$ mass-fraction distribution noted by Wolfgang \& Lopez (2015). We calculated $\tau_{\text {env }}$ by taking instantaneous mass-loss rates to predict planet survival lifetimes. Are the differences in $\tau_{\text {env }}$ sufficiently large relative to the duration of active mass-loss to leave an observable imprint 
on the planet population? To what extent would the planet composition distribution be altered over the course of Gyr timespans? In the next section, we turn to evaluating the evolution in the $\mathrm{H} / \mathrm{He}$ inventory of a larger synthetic population of planets.

\subsection{Sampling a Simulated Planet Population}

In the previous section, we illustrated the effect of planet mass-loss on the $f_{\text {env }}$ distribution using instantaneous diagnostics at early times. We now synthesize all the implementations done so far to test the cumulative ramifications on planets in the present day. The planet population observed today (e.g., by Kepler) is a combination of both the initial outcomes from planet formation, and the subsequent effect of planet evolution. In this section, we focus solely on the latter process, isolating how planet evolution would affect an arbitrary initial planet composition distribution.

We borrow the language of observational cosmology to express the effects of planet mass-loss evolution on the distribution, $g\left(M_{\text {core }}, f_{\text {env }}, a, t\right)$ of planet masses and compositions, at orbital separation, $a$, and time, $t$. The transfer function $T$ ( $\left.M_{\text {core }}, f_{\text {env, }} f_{\text {env }, 0}, a, t\right)$ encapsulates how the initial planet mass-composition distribution output by the planet formation process, $g\left(M_{\text {core }}, f_{\text {env }}, a, t=0\right)$, will be modified over time

$$
\begin{aligned}
& g\left(M_{\mathrm{p}}, f_{\mathrm{env}}, a, t\right) \\
& =\int T\left(M_{\text {core }}, f_{\text {env }}, f_{\text {env }, 0}, a, t\right) g\left(M_{\text {core }}, f_{\text {env }, 0}, a, t=0\right) d f_{\text {env }, 0} .
\end{aligned}
$$

Planet-mass loss causes planets to evolve toward lower envelope mass fractions (and lower total masses) over time.

We present in Figure 7 a snapshot in time (at 4.5 Gyr age) and orbital separations at $0.1,0.07$, and 0.25 au of the planet evolution transfer function, marginalized over $f_{\text {env, } 0}$. The distribution of initial planet properties, $g\left(M_{\text {core }}, f_{\text {env }, 0}, a, t=0\right)$, was chosen to be uniformly distributed in $\log M_{\mathrm{p}}-\log f_{\text {env }}$ space; any peaks and deserts in Figure 7 are due solely to the subsequent consequences of mass-loss evolution. We considered initial masses ranging from $1-100 M_{\oplus}$, and initial $f_{\text {env }, 0}$ from $0.001-0.90$. For each orbital separation, we evolved 1200 planets with randomly generated conditions for 4.5 Gyr. Figure 7 presents final mass-composition distribution of the simulated planets, which effectively represents the distortion of the initial $\log M_{\mathrm{p}}-\log f_{\text {env }}$ distribution due to planet evolution.

We analyze some observations of the 0.1 au diagram (large panel in Figure 7). There is an over-density in the planet masscomposition distribution at envelope fractions of $\approx 10^{-2.0}-10^{-1.5}$ and planet masses $\lesssim 20 M_{\oplus}$. Planets residing in this regime correspond to those with compositions close to the most "optimal" longest-lived envelope fractions (with highest $\left.\tau_{\text {env }}\right)$. This is the manifestation over the cumulative history of the planets of the non-monotonic behavior of $\tau_{\text {env }}$ with $f_{\text {env }}$ highlighted in Section 4.5).

The 0.1 au diagram in Figure 7 also displays a paucity of planets with masses $\lesssim 17 M_{\oplus}$ surviving at $4.5 \mathrm{Gyr}$ with $f_{\text {env }} \lesssim 10^{-3} \%$. This is due to the short envelope mass-loss timescales in this regime (because there is little $\mathrm{H} / \mathrm{He}$ to lose).

For planets with masses above $\sim 16 M_{\oplus}\left(10^{1.2}\right)$, the "distortion" of the original distribution $g\left(M_{\mathrm{p}}, f_{\text {env, }, 0}, a, t=0\right)$ is less significant compared to the low-mass regime and even less so for further orbital separations (compare the degree of distortion for the 0.07 and 0.25 au panels). Although there is an apparent dearth of planets with $f_{\text {env }} \lesssim 0.001$, this desert arises as a pure artifact due to the way in which we set the lower bound initial compositions $\left(f_{\text {env }} \geqslant 0.001\right)$. These results reflect the fact that planets in this regime are less likely to lose mass at this specific orbital distance, $F_{\mathrm{p}} \approx 100 F_{\oplus}$.

Planet interior structure and evolution may also lead to low mass $\left(M_{\mathrm{p}} \lesssim 10 M_{\oplus}\right)$ and high envelope mass fractions $\left(f_{\text {env }} \gtrsim 10 \%\right)$ being rare. This is visible in Figure 7 as an underdensity in the lower right-hand corner. This desert arises from the combined consequence of the massive mass-loss rates due to large planet cross-sectional area as well as the instability of MESA computations at even greater sizes (or $f_{\text {env }}$ ). In the latter cases, planets in this regime can be created, but are unable to be evolved past ages of $\sim 5-10 \mathrm{Myr}$. Simulations with extremely low mean densities such as these cause the $\mathrm{H} / \mathrm{He}$ envelopes to be "unbounded" over a very short period of time (even without mass-loss). The low- $M_{\mathrm{p}}-$ high- $f_{\text {env }}$ boundary in the lower right corner of Figure 7 corresponds to the same boundary in the upper left corner of Figure 2.

Finally, we recover the convergent behavior of evolution tracks, in which points (if imagined as vector fields) move toward the lower left, along a constant core mass curve (not shown in the figure). In particular, a range of initial $\% \mathrm{H} / \mathrm{He}$ values from $1 \%-10 \%$ ended up with a similar composition at 4.5 Gyr $\left(f_{\text {env }} \sim 0.8 \%\right)$.

Ultimately, the transfer function (Figure 7) is convolved with the initial distribution of planet properties (immediately after formation) to yield the current planet mass-composition distribution observed today. As a proof of concept, we sample from the mass distribution of radial velocity planets from the California Planet Search reported by Howard et al. (2010) to define a planet-mass distribution (still assuming a flat distribution of initial $\log f_{\text {env }}$ ). The resulting composition distribution of planets at $4.5 \mathrm{Gyr}$ is shown in Figure 8 . With this more realistic initial planet-mass distribution, we can see a distinct peak of simulated planet occurrence at about $1 \% \mathrm{H} / \mathrm{He}$ mass fraction. This result suggests that evolution via evaporation may partly explain the compositional distribution of subNeptune-sized planets found by Wolfgang \& Lopez (2015).

In the previous two sections, we have shown that photoevaporation effects may, in certain regimes, lead to a favored envelope mass fraction where the envelope mass-loss timescale is optimized. However, we have focused only on evolutionary processes, encapsulated in the transfer function. The eventuality of planet evolution is also strongly shaped by the choice of initial masses and compositions. While the initial planet distribution we assumed here is an oversimplification, it nonetheless provides an encouraging demonstration that evolutionary processes play a large role in sculpting the planets observed today.

\section{DISCUSSION}

\subsection{Insights into the Kepler Planet Population}

Planet interior structure and evolution calculations can provide insights into the observed distribution of Kepler planet properties. Several planet modelers have already noted features that photoevaporation could produce in the radius-flux distribution of close-in planets, specifically a declining occurrence of sub-Neptune-sized planets with increasing irradiation as vulnerable low-density planets lose their envelopes (e.g., Lecavelier Des Etangs 2007; Lopez et al. 2012), and an "occurrence valley" in the planet 

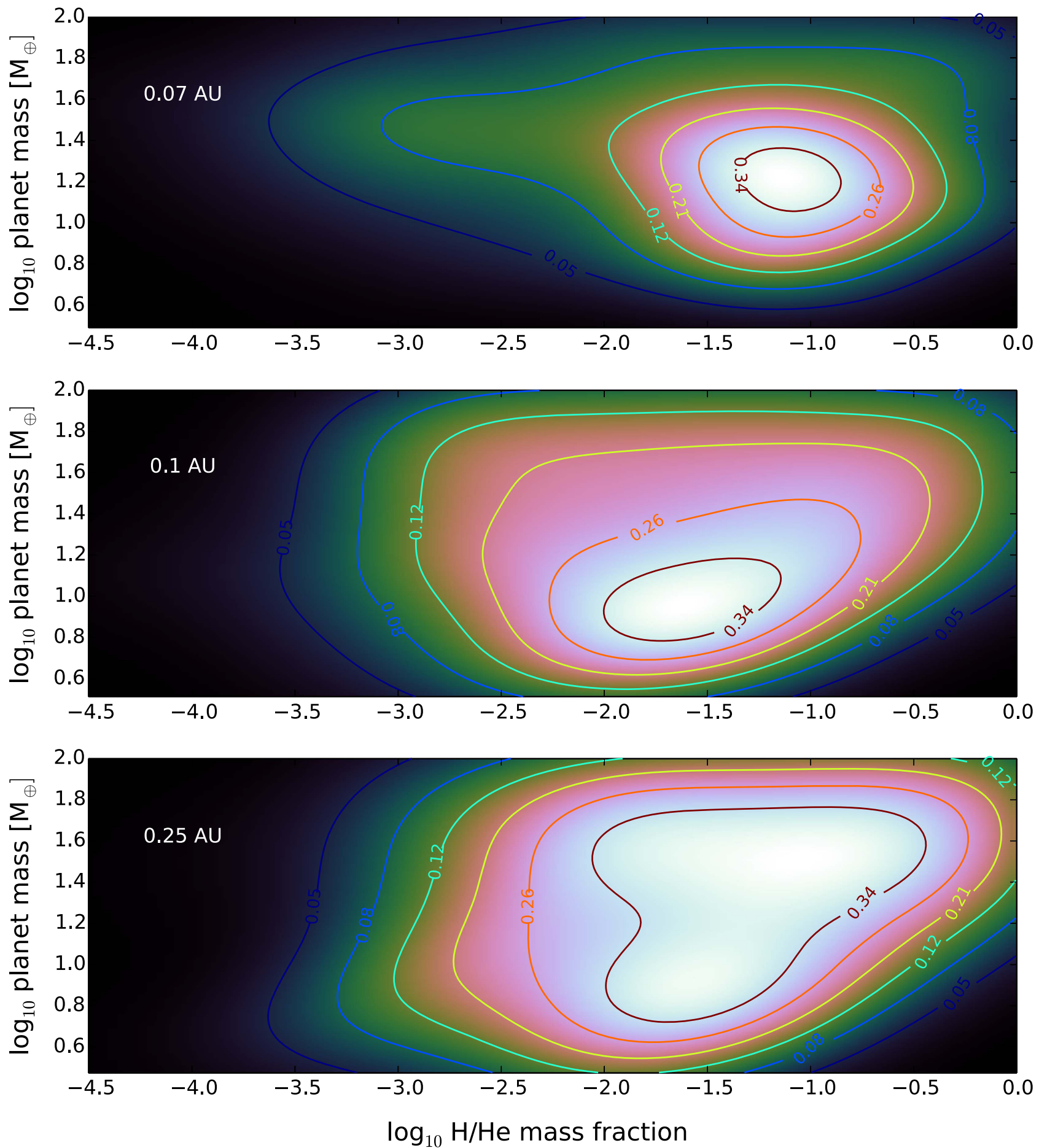

Figure 7. Final mass-composition distribution of simulated mass-losing planets at $4.5 \mathrm{Gyr}$ (top panel: $0.07 \mathrm{au}$, middle panel: $0.1 \mathrm{au}$, bottom panel: 0.25 au). The distribution of initial planet properties was chosen to be uniformly distributed in $\log M_{\mathrm{p}}-\log f_{\text {env }}$ space, with initial masses ranging from $1-100 M_{\oplus}$, and initial $f_{\text {env }, 0}$ from 0.001-0.95. We carried out calculations for 1200 simulated planets with randomly generated initial conditions for 4.5 Gyr. The final mass-composition distribution obtained from Gaussian kernel density estimation, is indicated by color-shading and contours, and represents a measure of the transfer function (at 4.5 Gyr), marginalized over $f_{\text {env }, 0}$.

distribution between $\sim 1.5 R_{\oplus}$ and $\sim 2.5 R_{\oplus}$ between the populations of planets that have retained their volatile envelopes and the population of remnant evaporated cores (e.g., Mordasini et al. 2012; Lopez \& Fortney 2013; Owen \& Wu 2013; Jin et al. 2014). Our planets' evolution simulations with MESA show good agreement (as expected) with these previously reported trends in the dividing line between complete and incomplete evaporation.
We have proposed yet another potential observable signature of evaporation in the close-in planet population, in Sections 4.5 and 4.6. Our simulations have hinted that the typical $\sim 1 \% \mathrm{H} /$ He mass fraction inferred from the Kepler radius distribution by Wolfgang \& Lopez (2015) could potentially be due to convergent evolution produced by evaporative planet massloss. This feature appears among the population of planets that suffer significant, but incomplete evaporation. With a toy 


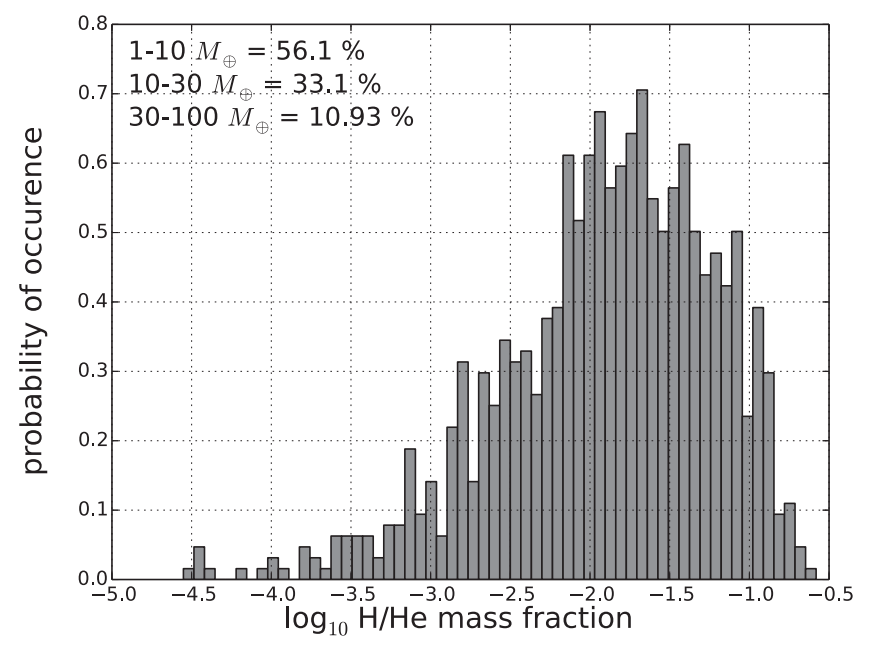

Figure 8. Synthetic planet probability-composition distribution at $4.5 \mathrm{Gyr}$ and 0.1 au. The initial $f_{\text {env }, 0}$ distribution assumed is flat in $\log f_{\text {env, } 0}$ (between $f_{\text {env, }, 0}$ of 0.001-0.90). The planet-mass distribution is sampled from the radial velocity mass distribution of Howard et al. (2010); the proportion of simulated planets within each mass bin is listed in the upper left corner of the plot. This 1D histogram is effectively Figure 7, marginalized over planet mass, weighted by the California Planet Search radial velocity mass distribution.

model for the initial planet mass-composition distribution, we have provided an illustrative proof of concept that this effect can have an observable influence in sculpting the eventual $f_{\text {env }}$ distribution at Gyr ages. Further work is needed to more fully quantify the effect of convergent photoevaporative evolution on the observed Kepler population, including models incorporating a more sophisticated treatment of planet mass-loss, a fully debiased joint radius-period distribution of Kepler planet candidates, and robust statistical methods to compare models and observations.

Ultimately, Kepler measured planet radii (transit depths) and it is to the distribution of planet radii that any model should be compared. In Figure 9 we present the distribution of planet radii associated with the synthetic planet population at 0.1 au in Figure 8, along with synthetic planet radius distributions at 0.07 and $0.25 \mathrm{au}$, generated following an identical approach. Our synthetic population shows the occurrence valley between evaporated cores and volatile-rich planets (between 1 and $2 R_{\oplus}$ ) that was found in previous theoretical works. The planet radius corresponding to the valley decreases with increasing orbital separation. As in the observed Kepler radius distribution from Petigura et al. (2013a), our synthetic radius distributions display an occurrence peak in the $2-2.83 R_{\oplus}$ bin (when logarithmic radius bins are employed). On the other hand, Petigura et al. (2013a) see a much steeper decline in planet occurrence between the $2-2.8 R_{\oplus}$ and $2.8-4 R_{\oplus}$ bins than is present in our synthetic population. We emphasize, however, that we assumed one of the simplest and broadest initial $M_{\mathrm{p}}-f_{\text {env }}$ distributions possible (flat in $\log f_{\text {env }}$, and no correlation between initial $M_{\mathrm{p}}$ and $f_{\text {env }}$ ); we made no attempt to tune the initial mass-composition distribution to fit the observed Kepler radius distribution. The steeper observed decline around $3 R_{\oplus}$ could be a consequence of a narrower distribution of initial compositions output from formation, evidence for a sub-population of water-rich planets, and/or correlations between initial planet mass and $f_{\text {env }}$. Indeed, planet formation models predict that correlations should exist between envelope mass and core mass (Bodenheimer \& Lissauer 2014;
Mordasini et al. 2014; Lee \& Chiang 2015). Further statistical studies linking Kepler data to models are warranted to better disentangle the effects of evolution and the outcomes of formation.

\subsection{Model Extension Opportunities}

We extended the MESA stellar evolution code to simulate $\mathrm{H} / \mathrm{He}$ envelopes surrounding low-mass planets in $1 \mathrm{D}$. The physics that we incorporated into MESA is not necessarily new; rather, we followed common assumptions and approaches employed in closed source (proprietary) 1D planet evolution codes that are in use in the field (e.g., Valencia et al. 2010; Lopez \& Fortney 2014; Kurokawa \& Nakamoto 2014, and Howe \& Burrows 2015). With our adaptations to MESA, there now exists a publicly available open source code to simulate $\mathrm{H} / \mathrm{He}$ planets down to a few Earth masses.

True planets are surely more complicated than the vanilla spherically symmetric, homogeneously layered scenarios simulated in this paper (and in prior works of Valencia et al. 2010; Kurokawa \& Nakamoto 2014; Lopez \& Fortney 2014; Howe \& Burrows 2015). These 1D simulations are, nonetheless, the current workhorses of exoplanet evolution studies, and help to provide context to more complex and computationally intensive models.

There are a number of physical processes that could be added to MESA in future work, to improve upon its capabilities to model planets.

These include a treatment of composition gradients within planet interiors (e.g., double diffusive convection of Nettelmann et al. 2015), using self-consistent model planet atmosphere grids to set the outer boundary conditions and planet cooling rates, (as in, e.g., Fortney et al. 2007), a more sophisticated treatment of atmospheric escape (including energy-limited scaling laws (Salz et al. 2016) and MHD effects), and finally the addition of a water EOS to facilitate the simulation of high mean molecular weight planetary envelopes.

It is our hope that the adaptations to MESA presented in this paper will help to provide the baseline groundwork for future applications of MESA to low-mass planets.

\section{SUMMARY \& CONCLUSIONS}

We summarize briefly below, the main outcomes and conclusions of this work.

(1) We implemented extensions to the MESA stellar evolution code that now permit MESA to simulate $\mathrm{H} /$ He envelopes surrounding planets down to a few Earth masses. These extensions include a thermophysical model for planet heavy-element interiors, energy-limited and radiation-recombination-limited mass-loss, and an improved atmospheric boundary condition.

(2) Coupled thermal and mass-loss evolution confirm that ultralow-density planets (with radii above $10 R_{\oplus}$ and masses below $30 M_{\oplus}$ ) can plausibly survive for multiple Gyr timescales, although over a narrower range of parameter space than that predicted by instantaneous mass-loss timescale criteria (Batygin \& Stevenson 2013).

(3) For mass-losing planets even at close orbital distances $\lesssim 0.1 \mathrm{au}$, radius is a proxy for the planet's current composition.

(4) Planet radii typically show very little hysteresis. The systematic error introduced by applying planet isochrones 

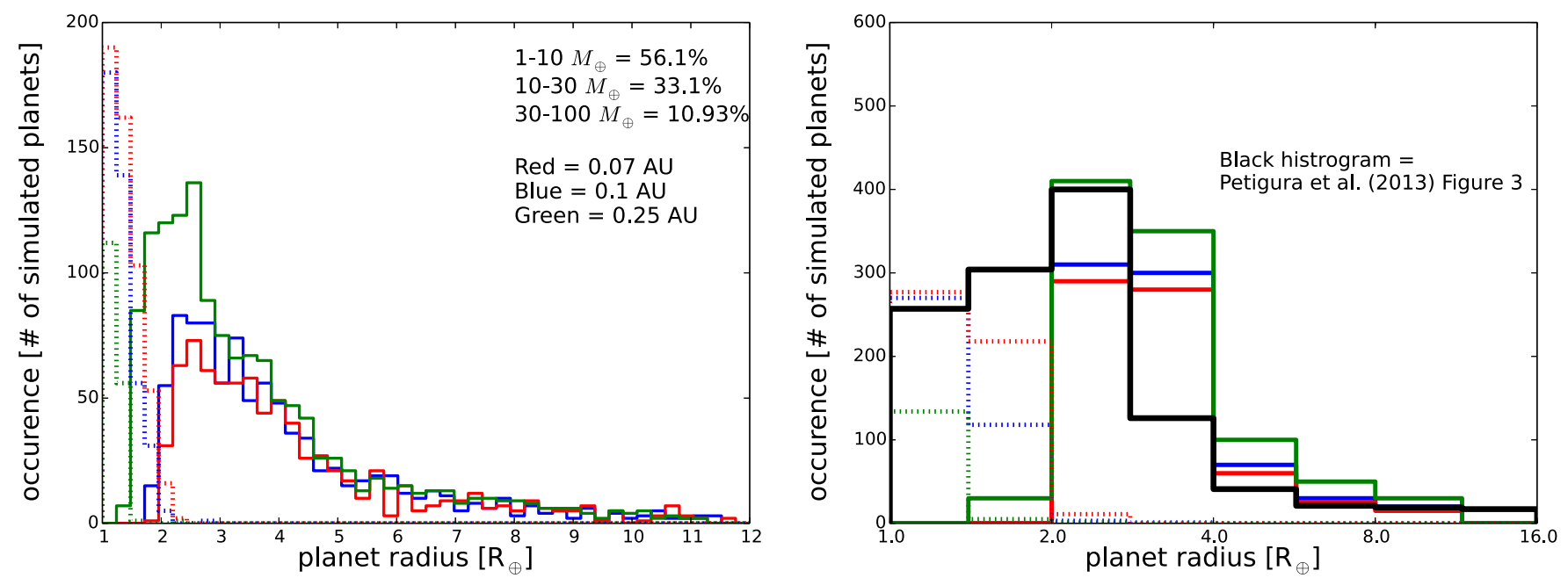

Figure 9. Synthetic planet radius distributions at $4.5 \mathrm{Gyr}$ on a linear radius scale (left) and logarithmic radius scale (right). The histogram colors indicate different orbital separations: 0.07 au (red), 0.10 au (blue), and 0.25 au (green). A total of 1200 planets was simulated at each orbital separation. The solid lines represent planet models that have retained some $\mathrm{H} / \mathrm{He}$ in their envelopes, while dotted lines correspond to evaporated planet cores. The blue histogram at 0.10 au corresponds to the same suite of simulations presented in Figure 8. The bottom panel is plotted for ease of comparison with the Kepler radius occurrence rates from Petigura et al. (2013a) (black step-curve).

calculated neglecting mass-loss to define a mapping from planet mass, composition, and age to radius for evaporating planets is typically small $(\lesssim 1 \%$ in planet radius), with the exception of models with very high envelope fractions.

(5) Planet envelope mass-loss timescales, $\tau_{\text {env }}$ vary nonmonotonically with $f_{\text {env }}$ (at fixed planet mass). In our simulations of young $(100 \mathrm{Myr})$ low-mass $\left(M_{\mathrm{p}} \lesssim 10 M_{\oplus}\right)$ planets with rocky cores, $\tau_{\text {env }}$ is maximized at $f_{\text {env }}=1 \%$ to $3 \%$. The resulting convergent evolution could potentially imprint itself on the close-in planet population as a preferred $\mathrm{H} / \mathrm{He}$ mass fraction of $\sim 1 \%$ (as inferred from the Kepler radius distribution by Wolfgang \& Lopez 2015)

With a succession of space-based exoplanet transit surveys on the horizon (K2, TESS, CHEOPS, and PLATO) combined with improving the resolution and stability of ground-based spectrographs (e.g., SPIRou, Keck SHREK, EXPRES, Carmenes, HPF, ESPRESSO, G-CLEF, and HiJaK), our purview of exoplanetary systems is bound to expand vastly in the years to come. For this reason, there is a need for a fast yet robust series of modeling and computational schemes to complement observational measurements. Recent years has seen a near simultaneous rise in the use of the stellar evolution code MESA and planetary mass-loss evolution studies. In this article, we provide a suite of basic planet models from which more complicated studies can be built; this is again eased by the open source nature of MESA. Looking ahead, we see this wonderful evolution code acting as a complement to more sophisticated 3D models to interpret the measurements of future space missions and exoplanetary surveys.

We thank Dr. James Owen for assisting us with the atmospheric boundary conditions and Prof. Phil Arras for providing us with helpful templates that enhanced the efficiency of simulating MESA planets. We also thank Dr. Eric Lopez for great suggestions for our manuscript. H.C. acknowledges the Undergraduate Research Opportunities Program (UROP) at Boston University for funding this research, while he was in residence at Caltech during the summer of 2014 and throughout the academic year. The authors thank Professors Philip Muirhead and Heather Knutson for facilitating the summer research. L.A.R. gratefully acknowledges support provided by NASA through the Hubble Fellowship Grant \#HF-51313 awarded by the Space Telescope Science Institute, which is operated by the Association of Universities for Research in Astronomy, Inc., for NASA, under contract NAS 5-26555. This work was performed in part under contract with the Jet Propulsion Laboratory (JPL) funded by NASA through the Sagan Fellowship Program executed by the NASA Exoplanet Science Institute. Most of the calculations have made use of H.C.'s MSI GE70 APACHE laptop, which was supported by his parents to pursue his interest in computational astrophysics and planetary science. In conclusion, we express our gratitude to the MESA code creators, Dr. Bill Paxton, and Professors Lars Bildsten and Frank Timmes, without whom this project would not have been possible.

\section{REFERENCES}

Baraffe, I., Chabrier, G., \& Barman, T. 2008, A\&A, 482, 315

Batalha, N. M., Rowe, J. F., Bryson, S. T., et al. 2013, ApJS, 204, 24 Batygin, K., \& Stevenson, D. J. 2013, ApJL, 769, L9

Becker, J. C., \& Batygin, K. 2013, ApJ, 778, 100

Bodenheimer, P., \& Lissauer, J. J. 2014, ApJ, 791, 103

Borucki, W. J., Koch, D. G., Basri, G., et al. 2011, ApJ, 728, 117

Burke, C. J., Bryson, S. T., Mullally, F., et al. 2014, ApJS, 210, 19

Burke, C. J., Christiansen, J. L., Mullally, F., et al. 2015, ApJ, 809, 8

Carter, J. A., Agol, E., Chaplin, W. J., et al. 2012, Sci, 337, 556

Chadney, J. M., Galand, M., Unruh, Y. C., Koskinen, T. T., \& Sanz-Forcada, J. 2015, Icar, 250, 357

Erkaev, N. V., Kulikov, Y. N., Lammer, H., et al. 2007, A\&A, 472, 329

Fortney, J. J., Marley, M. S., \& Barnes, J. W. 2007, ApJ, 659, 1661

Freedman, R. S., Lustig-Yaeger, J., Fortney, J. J., et al. 2014, ApJS, 214, 25 Freedman, R. S., Marley, M. S., \& Lodders, K. 2008, ApJS, 174, 504 Fressin, F., Torres, G., Charbonneau, D., et al. 2013, ApJ, 766, 81 Guillot, T. 2010, A\&A, 520, A27

Guillot, T., Chabrier, G., Gautier, D., \& Morel, P. 1995, ApJ, 450, 463 Han, E., Wang, S. X., Wright, J. T., et al. 2014, PASP, 126, 827

Hartmann, W. 2004, Moons and Planets (New York: Cengage Learning) Howard, A. W., Marcy, G. W., Bryson, S. T., et al. 2012, ApJS, 201, 15 Howard, A. W., Marcy, G. W., Johnson, J. A., et al. 2010, Sci, 330, 653 Howe, A. R., \& Burrows, A. 2015, ApJ, 808, 150 
Howe, A. R., Burrows, A., \& Verne, W. 2014, ApJ, 787, 173

Jackson, A. P., Davis, T. A., \& Wheatley, P. J. 2012, MNRAS, 422, 2024

Jackson, B., Jensen, E., Peacock, S., Arras, P., \& Penev, K. 2016, CeMDA, 126, 227ADS

Jin, S., Mordasini, C., Parmentier, V., et al. 2014, ApJ, 795, 65

Jontof-Hutter, D., Lissauer, J. J., Rowe, J. F., \& Fabrycky, D. C. 2014, ApJ, 785,15

Kasting, J. F., \& Pollack, J. B. 1983, Icar, 53, 479

Kurokawa, H., \& Nakamoto, T. 2014, ApJ, 783, 54

Lammer, H., Selsis, F., Ribas, I., et al. 2003, ApJL, 598, L121

Lecavelier Des Etangs, A. 2007, A\&A, 461, 1185

Lee, E. J., \& Chiang, E. 2015, ApJ, 811, 41

Lissauer, J. J., Fabrycky, D. C., Ford, E. B., et al. 2011, Natur, 470, 53

Lissauer, J. J., Jontof-Hutter, D., Rowe, J. F., et al. 2013, ApJ, 770, 131

Lopez, E. D., \& Fortney, J. J. 2013, ApJ, 776, 2

Lopez, E. D., \& Fortney, J. J. 2014, ApJ, 792, 1

Lopez, E. D., Fortney, J. J., \& Miller, N. 2012, ApJ, 761, 59

Luger, R., Barnes, R., Lopez, E., et al. 2015, AsBio, 15, 57

Marcy, G., Isaacson, H., Howard, A. W., et al. 2014, ApJS, 210, 20

Masuda, K. 2014, ApJ, 783, 53

Mcley, L., \& Soker, N. 2014, MNRAS, 445, 2492

Mordasini, C., Alibert, Y., Georgy, C., et al. 2012, A\&A, 547, A112

Mordasini, C., Klahr, H., Alibert, Y., Miller, N., \& Henning, T. 2014, A\&A, 566, A141

Mullally, F., Coughlin, J. L., Thompson, S. E., et al. 2015, ApJS, 217, 31

Murray-Clay, R. A., Chiang, E. I., \& Murray, N. 2009, ApJ, 693, 23

Nettelmann, N., Fortney, J. J., Kramm, U., \& Redmer, R. 2011, ApJ, 733, 2

Nettelmann, N., Fortney, J. J., Moore, K., \& Mankovich, C. 2015, MNRAS, 447,3422

Owen, J. E., \& Alvarez, M. A. 2016, ApJ, 816, 34

Owen, J. E., \& Jackson, A. P. 2012, MNRAS, 425, 2931

Owen, J. E., \& Wu, Y. 2013, ApJ, 775, 105
Owen, J. E., \& Wu, Y. 2016, ApJ, 817, 107

Paxton, B., Bildsten, L., Dotter, A., et al. 2011, ApJS, 192, 3

Paxton, B., Cantiello, M., Arras, P., et al. 2013, ApJS, 208, 4

Paxton, B., Marchant, P., Schwab, J., et al. 2015, ApJS, 220, 15

Perna, R., Duffell, P., Cantiello, M., \& MacFadyen, A. I. 2014, ApJ, 781, 119

Petigura, E. A., Howard, A. W., \& Marcy, G. W. 2013a, PNAS, 110, 19273

Petigura, E. A., Marcy, G. W., \& Howard, A. W. 2013b, ApJ, 770, 69

Ribas, I., Guinan, E. F., Güdel, M., \& Audard, M. 2005, ApJ, 622, 680

Rogers, L. A. 2015, ApJ, 801, 41

Rogers, L. A., Bodenheimer, P., Lissauer, J. J., \& Seager, S. 2011, ApJ, 738, 59

Rogers, L. A., \& Seager, S. 2010, ApJ, 712, 974

Rowe, J. F., Bryson, S. T., Marcy, G. W., et al. 2014, ApJ, 784, 45

Salz, M., Schneider, P. C., Czesla, S., \& Schmitt, J. H. M. M. 2016, A\&A, 585,2

Sanchis-Ojeda, R., Fabrycky, D. C., Winn, J. N., et al. 2012, Natur, 487, 449

Saumon, D., Chabrier, G., \& van Horn, H. M. 1995, ApJS, 99, 713

Tripathi, A., Kratter, K. M., Murray-Clay, R. A., \& Krumholz, M. R. 2015, ApJ, 808, 173

Valencia, D., Ikoma, M., Guillot, T., \& Nettelmann, N. 2010, A\&A, 516, A20

Valsecchi, F., Rappaport, S., Rasio, F. A., Marchant, P., \& Rogers, L. A. 2015, ApJ, 813, 101

Valsecchi, F., Rasio, F. A., \& Steffen, J. H. 2014, ApJL, 793, L3

Vidotto, A. A., Fares, R., Jardine, M., Moutou, C., \& Donati, J.-F. 2015, MNRAS, 449, 4117

Watson, A. J., Donahue, T. M., \& Walker, J. C. G. 1981, Icar, 48, 150

Weiss, L. M., \& Marcy, G. W. 2014, ApJL, 783, L6

Wolf, W. M., Bildsten, L., Brooks, J., \& Paxton, B. 2013, ApJ, 777, 136

Wolfgang, A., \& Lopez, E. 2015, ApJ, 806, 183

Wolfgang, A., Rogers, L. A., \& Ford, E. B. 2015, arXiv:1504.07557

Wu, Y., \& Lithwick, Y. 2013, ApJ, 763, 13 